\title{
Vertex Coalgebras, Coassociator, and Cocommutator Formulas
}

\author{
Florencia Orosz Hunziker and José I. Liberati \\ Ciem-FAMAF, Universidad Nacional de Córdoba, Ciudad Universitaria, 5000 Córdoba, Argentina \\ Correspondence should be addressed to Florencia Orosz Hunziker; o.flor.po@gmail.com
}

Received 23 September 2013; Accepted 15 December 2013; Published 2 March 2014

Academic Editor: Antonio M. Cegarra

Copyright (C) 2014 F. Orosz Hunziker and J. I. Liberati. This is an open access article distributed under the Creative Commons Attribution License, which permits unrestricted use, distribution, and reproduction in any medium, provided the original work is properly cited.

Based on the definition of vertex coalgebra introduced by Hubbard, 2009, we prove that this notion can be reformulated using coskew symmetry, coassociator and cocommutator formulas without restrictions on the grading. We also prove that a vertex coalgebra can be defined in terms of dual versions of the axioms of Lie conformal algebra and differential algebra.

\section{Introduction}

The notion of vertex coalgebra was introduced by Hubbard in [1] as a generalization of the notion of vertex operator coalgebra previously studied in [2-4]. Many of the properties described in these works are in some sense dual to the properties satisfied by vertex algebras. Since the original definition of vertex algebra was introduced by Borcherds [5] in the 1980s, several reformulations have been studied. A vertex algebra can be defined using Lie algebra-type axioms or it can be seen as a generalization of a commutative and associative algebra with unit, focusing on the commutator or associator formulas. These formulations are introduced and thoroughly studied in [6-8]. A vertex algebra can also be defined as the deformation of a Poisson vertex algebra, namely, a Lie conformal algebra and a left symmetric differential algebra with unit satisfying certain compatibilities, as described in [9]. These different approaches engender several equivalent definitions of vertex algebra based on different axioms. Our goal is to prove that these approaches can be, in some sense, dualized to obtain equivalent definitions of vertex coalgebra.

The study of these approaches is not automatic in the case of vertex coalgebras as there might be axioms in the definition of vertex algebra that do not make sense in their dual version. For instance, axioms such as "weak commutativity" and "weak associativity" do not make sense unless we require the use of grading on a vertex coalgebra (see [1]). In [10], the authors show that with a coefficient approach, the Jacobi identity can be proven to follow from the commutator and associator formulas. Based on that idea, we first obtain a reformulation of the original definition of vertex coalgebra analogous to the original definition of vertex algebra introduced by Borcherds [5]. Then, we prove that the original definition of vertex coalgebra can be reformulated in three equivalent definitions: the first based on the coassociator and coskew symmetry formulas, the second based on the cocommutator formula, and the third based on dual versions of the axioms of Lie conformal algebra and differential algebra, following the ideas developed in [9].

In the next section we introduce basic definitions and results, as well as the original definition of vertex coalgebra and its basic properties. In Section 3, following the idea presented in [10], we prove that the coefficient version of the co-Jacobi identity (called co-Borcherds formula) can be deduced from weaker versions of this formula. In Section 4 we prove that the definition of vertex coalgebra can be reformulated based on coskew symmetry, coassociator, cocommutator, and $D^{\star}$ formulas. Finally, in Section 5, we define a vertex coalgebra in terms of axioms regarding the $n$ coproducts for $n \geq-1$.

\section{Basic Properties and the Original Definition}

We begin by introducing some basic definitions and results from the calculus of formal variables. A more thorough description of the concepts defined in this section, as well as the proofs of the following results, can be found in $[6,7,11]$. 
We will consider $x, y$, and $z$ commuting formal variables and define the formal $\delta$-function to be

$$
\delta(x, y)=\sum_{n \in \mathbb{Z}} x^{n} y^{-n-1}
$$

Given an integer $n$, we define

$$
(x \pm y)^{n}=\sum_{k \geq 0}\left(\begin{array}{l}
n \\
k
\end{array}\right) x^{n-k}( \pm y)^{k},
$$

where $\left(\begin{array}{l}n \\ k\end{array}\right)=(n(n-1) \cdots(n-k+1)) / k$ ! for $n \in \mathbb{Z}$ and $k \geq 0$. Note that $\delta(x-y, z)$ is a formal power series in nonnegative powers of $y$. The formal residue "Res $x$ " of a series $v(x)=$ $\sum_{n \in \mathbb{Z}} v_{n} x^{n}$ refers to the coefficient of the negative first power; that is,

$$
\operatorname{Res}_{x} v(x)=v_{-1} .
$$

We will also use Taylor's Theorem, which states that given a $\mathbb{C}$-vector space $V$ and a formal series $f(x) \in V\left[\left[x, x^{-1}\right]\right]$, we have

$$
e^{y(d / d x)} f(x)=f(x+y) .
$$

We define the linear map $T: V \otimes V \rightarrow V \otimes V$ to be the transposition operator described by $T(v \otimes u)=u \otimes v$ for all $u, v \in V$.

Next, we enumerate basic properties of the formal series $\delta$ that we will need throughout this work:

$$
\begin{aligned}
& \text { (a) } \operatorname{Res}_{y} \delta(x, y)=\operatorname{Res}_{y} \delta(y, x)=1 . \\
& \text { (b) }(x-y) \delta(x, y)=0 . \\
& \text { (c) } \delta(x-y, z)=\delta(z+y, x) . \\
& \text { (d) } \delta(x-y, z)-\delta(-y+x, z)=\delta(x-z, y) .
\end{aligned}
$$

We will also make use of the following result (cf. Proposition 2.1.1 [11]).

Given a formal Laurent series $X(x, y) \in(\operatorname{Hom}(V, W))[[x$, $\left.\left.x^{-1}, y, y^{-1}\right]\right]$ with coefficients which are homomorphisms from a vector space $V$ to a vector space $W$, if $\lim _{x \rightarrow y} X(x, y)$ exists (i.e., when $X(x, y)$ applied to any element of $V$ setting $x=y$ leads to only finite sums in $W$ ), we have

$$
\delta(x, y) X(x, y)=\delta(x, y) X(y, y) .
$$

In particular, we have

$$
\operatorname{Res}_{x} \delta(x, y) X(x, y)=X(y, y) .
$$

Now that we have listed the results that we will need, let us recall the original definition of vertex coalgebra introduced in [1].

Definition 1. A vertex coalgebra consists of a $\mathbb{C}$-vector space $V$, together with linear maps

$$
\begin{gathered}
\lambda(x): V \longrightarrow(V \otimes V)\left[\left[x, x^{-1}\right]\right], \\
c: V \longrightarrow \mathbb{C}
\end{gathered}
$$

called the coproduct and covacuum map, respectively, satisfying the following axioms for all $v \in V$. (i) Left counit:

$$
(c \otimes \operatorname{Id}) \lambda(x) v=v .
$$

(ii) Cocreation:

$(\operatorname{Id} \otimes c) \wedge(x) v \in V[[x]],\left.\quad(\operatorname{Id} \otimes c) \wedge(x) v\right|_{x=0}=v$.

(iii) Truncation:

$$
\wedge(x) v \in(V \otimes V)\left(\left(x^{-1}\right)\right) .
$$

(iv) Co-Jacobi identity:

$$
\begin{aligned}
\delta(x-z, y)(\lambda(z) \otimes \mathrm{Id}) \wedge(y) & \\
= & \delta(x-y, z)(\operatorname{Id} \otimes \wedge(y)) \wedge(x) \\
& \quad-\delta(-y+x, z)(T \otimes \operatorname{Id})(\operatorname{Id} \otimes \wedge(x)) \wedge(y) .
\end{aligned}
$$

Remarks 1. (a) The operator $\lambda(x)$ is linear so that, for example, $(\mathrm{Id} \otimes \lambda(y))$ acting on the coefficients of $\lambda(x) v \in$ $(V \otimes V)\left[\left[x, x^{-1}\right]\right]$ is well defined.

(b) Note that when each expression is applied to any element of $V$, the coefficient of each monomial in the formal variables is a finite sum due to the truncation condition (14).

As an immediate result, we have the following.

Proposition 2. Let $(V, \lambda(x), c)$ be a vertex coalgebra as in Definition 1. Then, the following formulas hold.

(1) Cocommutator formula:

$$
\begin{aligned}
\operatorname{Res}_{z} \delta(x-z, y)(\lambda(z) \otimes I d) \wedge(y) \\
=(\operatorname{Id} \otimes \wedge(y)) \wedge(x)-(T \otimes I d) \\
\quad \times(\operatorname{Id} \otimes \wedge(x)) \wedge(y) .
\end{aligned}
$$

(2) Coassociator formula:

$$
\begin{aligned}
(\lambda(z) \otimes \mathrm{Id}) \wedge(y)= & (\operatorname{Id} \otimes \wedge(y)) \wedge(z+y) \\
& -\operatorname{Res}_{x} \delta(-y+x, z)(T \otimes \mathrm{Id}) \\
& \times(\operatorname{Id} \otimes \lambda(x)) \wedge(y) .
\end{aligned}
$$

Proof. (1) Taking Res $_{z}$ to the co-Jacobi identity (15), we obtain

$$
\begin{aligned}
\operatorname{Res}_{z} \delta & (x-z, y)(\wedge(z) \otimes \mathrm{Id}) \wedge(y) \\
= & \operatorname{Res}_{z} \delta(x-y, z)(\operatorname{Id} \otimes \wedge(y)) \wedge(x) \\
& \quad-\operatorname{Res}_{z} \delta(-y+x, z)(T \otimes \mathrm{Id})(\operatorname{Id} \otimes \wedge(x)) \wedge(y) .
\end{aligned}
$$

Using (5) twice on the right hand side of (18) we obtain (16).

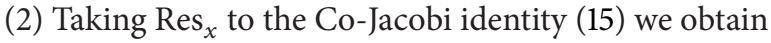

$$
\begin{aligned}
\operatorname{Res}_{x} \delta(x-z, y)(\lambda(z) \otimes \operatorname{Id}) \wedge(y) \\
=\operatorname{Res}_{x} \delta(x-y, z)(\operatorname{Id} \otimes \wedge(y)) \wedge(x) \\
\quad-\operatorname{Res}_{x} \delta(-y+x, z)(T \otimes \operatorname{Id})(\operatorname{Id} \otimes \wedge(x)) \wedge(y) .
\end{aligned}
$$


Using (5) and (7) on the left hand side of (19) we get

$$
\begin{aligned}
(\wedge(z) \otimes \mathrm{Id}) \wedge(y)= & \operatorname{Res}_{x} \delta(x-y, z)(\operatorname{Id} \otimes \wedge(y)) \wedge(x) \\
& -\operatorname{Res}_{x} \delta(-y+x, z)(T \otimes \mathrm{Id}) \\
& \times(\operatorname{Id} \otimes \wedge(x)) \wedge(y) .
\end{aligned}
$$

Finally, using (7) and (10) on the first term of the right hand side of (20) we obtain (17).

As pointed in [1], it is natural to question the effect of applying a formal derivative, $d / d x$, to the comultiplication operator. In order to study that effect we need to introduce a map. Given a vertex coalgebra $V$, we define the linear map $D^{\star}: V \rightarrow V$ as

$$
D^{\star}=\operatorname{Res}_{x} x^{-2}(\operatorname{Id} \otimes c) \wedge(x) .
$$

The following proposition obtained in [1] describes the main properties of the operator $D^{\star}$ and its relationship to the formal derivative.

Proposition 3. The map $D^{\star}$ satisfies the following properties:

(1)

$$
\left(D^{\star} \otimes \mathrm{Id}\right) \wedge(x)=\frac{d}{d x} \wedge(x)
$$

(2)

$$
\left(e^{z D^{\star}} \otimes \mathrm{Id}\right) \wedge(x)=\wedge(x+z)
$$

(3)

$$
e^{z D^{\star}}=(\operatorname{Id} \otimes c) \wedge(z)
$$

(4) coskew symmetry:

$$
T \wedge(x)=\curlywedge(-x) e^{x D^{*}}
$$

(5)

$$
\frac{d}{d x} \wedge(x)=\wedge(x) D^{\star}-\left(\operatorname{Id} \otimes D^{\star}\right) \wedge(x)
$$

(6)

$$
\left(\mathrm{Id} \otimes e^{-z D^{\star}}\right) \wedge(x) e^{z D^{\star}}=\curlywedge(x+z) .
$$

We consider the following expansion of the map $\lambda(x)$ :

$$
\lambda(x) v=\sum_{n \in \mathbb{Z}} \Delta_{n}(v) x^{-n-1}
$$

where $\Delta_{n}: V \rightarrow V \otimes V$ is the coefficient of $x^{-n-1}$ in the series $\lambda(x)$. If we look for the coefficient of $x^{-p^{-1}} y^{-q^{-1}} z^{-r-1}$ in the co-Jacobi identity (15) we obtain what we will call the co-Borcherds identity:

$$
\begin{aligned}
\sum_{i \geq 0}\left(\begin{array}{c}
p \\
i
\end{array}\right)\left(\Delta_{r+i} \otimes \mathrm{Id}\right) \Delta_{p+q-i} \\
=\sum_{i \geq 0}(-1)^{i}\left(\begin{array}{c}
r \\
i
\end{array}\right)\left[\left(\operatorname{Id} \otimes \Delta_{q+i}\right) \Delta_{p+r-i}\right. \\
\left.\quad-(-1)^{r}(T \otimes \operatorname{Id})\left(\operatorname{Id} \otimes \Delta_{p+i}\right) \Delta_{q+r-i}\right] .
\end{aligned}
$$

We can rewrite Definition 1 considering coefficients. With this approach we obtain the following definition of a vertex coalgebra, evidently equivalent to the one previously introduced.

Definition 4. A vertex coalgebra is a $\mathbb{C}$-vector space $V$ endowed with a family of linear coproducts indexed by $n \in \mathbb{Z}$ and a linear map $c$ :

$$
\begin{gathered}
\Delta_{n}: V \longrightarrow V \otimes V, \\
c: V \longrightarrow \mathbb{C}
\end{gathered}
$$

satisfying the following axioms.

(i) Left counit:

$$
\begin{aligned}
& (c \otimes \text { Id }) \Delta_{n}=\text { Id } \quad \text { if } n=-1, \\
& (c \otimes \text { Id }) \Delta_{n}=0 \quad \text { if } n \neq-1 .
\end{aligned}
$$

(ii) Cocreation:

$$
\begin{aligned}
& (\operatorname{Id} \otimes c) \Delta_{n}=0 \quad \text { if } n \geq 0, \\
& (\operatorname{Id} \otimes c) \Delta_{-1}=\text { Id. }
\end{aligned}
$$

(iii) Truncation: for each $v \in V$ there exists $N$ such that

$$
\Delta_{n} v=0 \quad \forall n \leq N
$$

(iv) Co-Borcherds identity: for all $p, q, r \in \mathbb{Z}$,

$$
\begin{aligned}
\sum_{i \geq 0}\left(\begin{array}{c}
p \\
i
\end{array}\right)\left(\Delta_{r+i} \otimes \mathrm{Id}\right) \Delta_{p+q-i} \\
=\sum_{i \geq 0}(-1)^{i}\left(\begin{array}{c}
r \\
i
\end{array}\right)\left[\left(\mathrm{Id} \otimes \Delta_{q+i}\right) \Delta_{p+r-i}\right. \\
\left.-(-1)^{r}(T \otimes \mathrm{Id})\left(\mathrm{Id} \otimes \Delta_{p+i}\right) \Delta_{q+r-i}\right] .
\end{aligned}
$$

Under this coefficient approach the definition of $D^{\star}$ corresponds to the linear map

$$
\begin{gathered}
D^{\star}: V \longrightarrow V, \\
D^{\star}=(\operatorname{Id} \otimes c) \Delta_{-2} .
\end{gathered}
$$


Proposition 5. Let $V$ be a vertex coalgebra as in Definition 4 and $D^{\star}$ defined as in (35). Then, the following properties hold.

(1) For all $q \in \mathbb{Z}$,

$$
\left(D^{\star} \otimes \mathrm{Id}\right) \Delta_{q}=-q \Delta_{q-1} .
$$

(2) If we denote $D^{\star(i)}=(1 / i !)\left(D^{\star}\right)^{i}$ for $i \geq 0$, then

$$
D^{\star(i)}=(\mathrm{Id} \otimes c) \Delta_{-1-i} .
$$

(3) The operator $D^{\star}$ is locally nilpotent. Namely, for each $v \in V$ there exists $N$ such that $\left(D^{\star}\right)^{n}(v)=$ 0 for all $n \geq N$.

Proof. (1) If we take the generating function in $x$ of (36) we obtain

$$
\left(D^{\star} \otimes \mathrm{Id}\right) \wedge(x)=\frac{d}{d x} \wedge(x)
$$

Therefore, (36) is just the coefficient formulation of (22).

(2) It is easy to see that (37) is merely the coefficient formulation of (24).

(3) The proof follows from (37) together with (33).

Remarks 2. Note that for the proof of (1) and (2) in the previous proposition we only used the definition of $D^{\star}$ together with axioms (22) and (24).

Manipulating expressions that involve coefficients we will get coefficient versions of the cocommutator formula (16), the coassociator formula (17), and the coskew symmetry formula (25) based on Definition 4. These formulas will be axioms in the two definitions of vertex coalgebra that we will introduce in Section 4.

Proposition 6. Let $V$ be a vertex coalgebra as in Definition 4. Then, the following formulas hold for every $p, q, r \in \mathbb{Z}$.

(1) Coefficient cocommutator formula:

$$
\begin{aligned}
& \sum_{i \geq 0}\left(\begin{array}{c}
p \\
i
\end{array}\right)\left(\Delta_{i} \otimes \mathrm{Id}\right) \Delta_{p+q-i} \\
& \quad=\left(\operatorname{Id} \otimes \Delta_{q}\right) \Delta_{p}-(T \otimes I d)\left(\operatorname{Id} \otimes \Delta_{p}\right) \Delta_{q} .
\end{aligned}
$$

(2) Coefficient coassociator formula:

$$
\begin{aligned}
\left(\Delta_{r} \otimes \mathrm{Id}\right) \Delta_{q}=\sum_{i \geq 0} & (-1)^{i}\left(\begin{array}{c}
r \\
i
\end{array}\right) \\
\times & {\left[\left(\operatorname{Id} \otimes \Delta_{q+i}\right) \Delta_{r-i}-(-1)^{r}\right.} \\
& \left.\times(T \otimes \mathrm{Id})\left(\operatorname{Id} \otimes \Delta_{i}\right) \Delta_{q+r-i}\right] .
\end{aligned}
$$

(3) Coefficient coskew symmetry:

$$
T \Delta_{r}=\sum_{i \geq 0}(-1)^{r+1+i} \Delta_{r+i} D^{\star(i)}
$$

Proof. The proof follows from the fact that Definitions 1 and 4 are equivalent. Formulas (39), (40), and (41) are merely the coefficient formulation of formulas (16), (17), and (25) respectively.

\section{Structure of the Co-Borcherds Identity}

Following the idea presented in [10], we want to prove that the Co-Borcherds identity can be deduced from the cocommutator and the coassociator formulas. For that reason, we introduce a few auxiliary formulas:

$$
\begin{aligned}
\mathrm{CB}_{1}(p, q, r):= & \sum_{i \geq 0}\left(\begin{array}{c}
p \\
i
\end{array}\right)\left(\Delta_{r+i} \otimes \mathrm{Id}\right) \Delta_{p+q-i} \\
\mathrm{CB}_{2}(p, q, r):= & \sum_{i \geq 0}(-1)^{i}\left(\begin{array}{l}
r \\
i
\end{array}\right)\left(\mathrm{Id} \otimes \Delta_{q+i}\right) \Delta_{p+r-i}, \\
\mathrm{CB}_{3}(p, q, r):= & \sum_{i \geq 0}(-1)^{i+r}\left(\begin{array}{l}
r \\
i
\end{array}\right) \\
& \quad \times(T \otimes \mathrm{Id})\left(\mathrm{Id} \otimes \Delta_{p+i}\right) \Delta_{q+r-i} .
\end{aligned}
$$

Note that with the notation introduced above, the coBorcherds identity (34) for the indices $p, q, r \in \mathbb{Z}$ corresponds to

$$
\mathrm{CB}_{1}(p, q, r)=\mathrm{CB}_{2}(p, q, r)-\mathrm{CB}_{3}(p, q, r) .
$$

We have the following formulas which are analogous to the results obtained in Section 3.2 [10].

Proposition 7. For all $p, q, r \in \mathbb{Z}$,

$$
C B_{j}(p+1, q, r)=C B_{j}(p, q+1, r)+C B_{j}(p, q, r+1),
$$

for $j=1,2,3$.

Proof. Note that the co-Borcherds identity (34) is the coefficient version of the co-Jacobi identity (15). Therefore, it is clear that if we consider

$$
\begin{gathered}
G_{1}(x, y, z):=\delta(x-z, y)(\lambda(z) \otimes \operatorname{Id}) \wedge(y), \\
G_{2}(x, y, z):=\delta(x-y, z)(\operatorname{Id} \otimes \wedge(y)) \wedge(x), \\
G_{3}(x, y, z):=\delta(-y+x, z)(T \otimes \operatorname{Id})(\operatorname{Id} \otimes \wedge(x)) \wedge(y),
\end{gathered}
$$

then, for $j=1,2,3$,

$$
\mathrm{CB}_{j}(p, q, r)=\operatorname{Res}_{x, y, z} x^{p} y^{q} z^{r} G_{j}(x, y, z) .
$$

We want to prove that for $j=1,2,3$,

$$
\mathrm{CB}_{j}(p+1, q, r)-\mathrm{CB}_{j}(p, q+1, r)-\mathrm{CB}_{j}(p, q, r+1)=0 .
$$

Note that (47) is equivalent to

$$
\begin{gathered}
\operatorname{Res}_{x, y, z}\left(x^{p+1} y^{q} z^{r} G_{j}(x, y, z)-x^{p} y^{q+1} z^{r} G_{j}(x, y, z)\right. \\
\left.-x^{p} y^{q} z^{r+1} G_{j}(x, y, z)\right) \\
=\operatorname{Res}_{x, y, z}\left(x^{p} y^{q} z^{r}(x-y-z) G_{j}(x, y, z)\right)=0,
\end{gathered}
$$

for $j=1,2,3$, which holds due to (6). 
Using (43) and Proposition 7, it is easy to prove the following result.

Proposition 8. The co-Borcherds identities for two of the indices $(p+1, q, r),(p, q+1, r)$, and $(p, q, r+1)$ imply the coBorcherds identity for the other index.

Now, we can prove the following statement.

Theorem 9. The co-Borcherds identities for all $p$ and $q$ with fixed $r$ and for all $q$ and $r$ with fixed $p$ imply the co-Borcherds identity for all $p, q$, and $r$.

Proof. We assume the co-Borcherds identity for all $p$ and $q$ with $r_{0}$ fixed and for all $q$ and $r$ with $p_{0}$ fixed. Using Proposition 8 we have that the co-Borcherds identity also holds for $\left(p, q, r_{0}+1\right)$ for all $p, q \in \mathbb{Z}$. Using that the coBorcherds identity holds for indices $\left(p, q+1, r_{0}+1\right)$ and $\left(p+1, q, r_{0}+1\right)$ together with Proposition 8 , we obtain that the co-Borcherds identity also holds for the indices $\left(p, q, r_{0}+2\right)$ for all $p, q \in \mathbb{Z}$. Inductively we obtain that the co-Borcherds identity holds for $\left(p, q, r_{0}+n\right)$ for all $n \in \mathbb{N}$ for every $p, q \in \mathbb{Z}$.

Analogously, we can prove that the co-Borcherds identity also holds for $\left(p_{0}+1, q, r\right)$ for all $q, r, \in \mathbb{Z}$. Inductively we obtain that the co-Borcherds identity holds for the indices $\left(p_{0}+n, q, r\right)$ for all $n \in \mathbb{N}$ for every $q, r \in \mathbb{Z}$. Thus, it is clear that the co-Borcherds identity holds for $(p, q, r)$ as long as $p \geq p_{0}$ or $r \geq r_{0}$.

Now, we need to analyze the case in which $p<p_{0}$ and $r<r_{0}$. First, we note that the co-Borcherds identity holds for indices $\left(p_{0}, s, r_{0}-1\right)$ and $\left(p_{0}-1, s, r_{0}\right)$ for all $s \in \mathbb{Z}$. Using Proposition 8 those identities imply the co-Borcherds identity for the index $\left(p_{0}-1, s+1, r_{0}-1\right)$ for all $s \in \mathbb{Z}$. Again, using that the co-Borcherds identity holds for indices $\left(p_{0}-1, s+1, r_{0}-1\right)$ and $\left(p_{0}-2, s+1, r_{0}\right)$ we obtain that coBorcherds identity holds for the index $\left(p_{0}-2, s+2, r_{0}-1\right)$. Inductively, we obtain that the co-Borcherds identity holds for indices $\left(p_{0}-n, s+n, r_{0}-1\right)$ for all $n \geq 0$. As this identity holds for all $s \in \mathbb{Z}$, we obtain that the co-Borcherds identity holds for indices $\left(p_{0}-n, s, r_{0}-1\right)$ for all $n \geq 0$ and $s \in \mathbb{Z}$.

Next, the fact that the co-Borcherds identity holds for indices $\left(p_{0}-1, l, r_{0}-1\right)$ and $\left(p_{0}, l, r_{0}-2\right)$ for all $l \in \mathbb{Z}$ implies that the co-Borcherds identity holds for the index $\left(p_{0}-1, l, r_{0}-2\right)$ for all $l \in \mathbb{Z}$. Inductively, we obtain that the co-Borcherds identity holds for indices $\left(p_{0}-1, s, r_{0}-n\right)$ for all $n \geq 0$ for every $s \in \mathbb{Z}$. Thus, we have proved that if the coBorcherds identity holds for indices $(p, q, r)$ as long as $p \geq p_{0}$ or $r \geq r_{0}$, then it also holds for $(p, q, r)$ as long as $p \geq p_{0}-1$ or $r \geq r_{0}-1$. Continuing with this procedure, we inductively obtain that the co-Borcherds identity holds for every index $(p, q, r)$, finishing the proof.

\section{Two Equivalent Definitions}

After studying the structure of the co-Borcherds identity, we will prove that we can reformulate the definition of a vertex coalgebra focusing on either the coassociator formula or the cocommutator formula.
Definition 10. A vertex coalgebra consists of a $\mathbb{C}$-vector space $V$, together with linear maps

$$
\begin{aligned}
& \wedge(x): V \longrightarrow(V \otimes V)\left(\left(x^{-1}\right)\right), \\
& c: V \longrightarrow \mathbb{C}, \\
& D^{\star}: V \longrightarrow V
\end{aligned}
$$

satisfying the following axioms for all $v \in V$.

(i) Left counit:

$$
(c \otimes \mathrm{Id}) \wedge(x) v=v .
$$

(ii) Cocreation:

$$
e^{x D^{\star}}=(\operatorname{Id} \otimes c) \wedge(x) .
$$

(iii) $D^{\star}$ formula:

$$
\frac{d}{d x} \wedge(x)=\left(D^{\star} \otimes \mathrm{Id}\right) \wedge(x) .
$$

(iv) Coskew symmetry:

$$
T \wedge(x)=\wedge(-x) e^{x D^{*}} .
$$

(v) Coassociator formula:

$$
\begin{aligned}
(\wedge(z) & \otimes \mathrm{Id}) \wedge(y) \\
= & (\mathrm{Id} \otimes \wedge(y)) \wedge(z+y)-\operatorname{Res}_{x} \delta(-y+x, z) \\
\quad & \times(T \otimes \operatorname{Id})(\operatorname{Id} \otimes \wedge(x)) \wedge(y) .
\end{aligned}
$$

Remarks 3. (a) Note that (51) trivially implies (13). That is, Definition 10 implies that for all $v \in V$,

$$
(\operatorname{Id} \otimes c) \wedge(x) v \in V[[x]],\left.\quad(\operatorname{Id} \otimes c) \wedge(x) v\right|_{x=0}=v .
$$

(b) Note that (51) implies that the map $D^{\star}$ is the map defined in (21). In fact, if we take the coefficient of $x^{1}$ in (51), we get $D^{\star}=(\operatorname{Id} \otimes c) \Delta_{-2}$.

(c) Using Proposition 3(3), it is clear that (51) is also satisfied under the conditions of Definition 1.

Hence, $D^{\star}$ is the map we introduced earlier and the conditions regarding the map $c$ do not essentially differ from the properties described in the first definitions. We can now state one of our main results.

\section{Theorem 11. Definition 10 is equivalent to Definition 1.}

In [1] (cf. Proposition 3), it is proved that Definition 1 implies cocreation (24), coskew symmetry (25), and the $D^{\star}$ formula (22). In Proposition 2(2) we proved that the coassociator formula (54) follows from Definition 1. Therefore, it is clear that the conditions of Definition 10 are satisfied under the axioms of Definition 1. In order to prove the other implication, we first need to prove some results. 
Proposition 12. The coskew symmetry formula (53) and the $D^{\star}$ formula (52) imply that the map $D^{\star}$ satisfies the following properties.

(1) $D^{\star}$-bracket formula:

$$
\frac{d}{d x} \wedge(x)=\wedge(x) D^{\star}-\left(\operatorname{Id} \otimes D^{\star}\right) \wedge(x) .
$$

(2) Conjugation formula:

$$
\left(\mathrm{Id} \otimes e^{-z D^{\star}}\right) \wedge(x) e^{z D^{\star}}=\wedge(x+z) .
$$

Proof. (1) Using (53), the product rule, (52), and the fact that $T\left(D^{\star} \otimes \mathrm{Id}\right)=\left(\mathrm{Id} \otimes D^{\star}\right) T$ and reapplying (53), we have

$$
\begin{aligned}
\frac{d}{d x} \wedge(x) & =\frac{d}{d x}\left(T \wedge(-x) e^{x D^{\star}}\right) \\
& =-T\left(D^{\star} \otimes \operatorname{Id}\right) \wedge(-x) e^{x D^{\star}}+T \wedge(-x) e^{x D^{\star}} D^{\star} \\
& =-\left(\operatorname{Id} \otimes D^{\star}\right) \wedge(x)+\wedge(x) D^{\star} .
\end{aligned}
$$

(2) Exponentiating the $D^{\star}$-bracket formula (56) and applying Taylor's Theorem (4) we obtain (57).

Our goal is to prove that the axioms of Definition $10 \mathrm{imply}$ the co-Jacobi identity (15). We begin by proving that the cocommutator formula (16) holds.

Lemma 13. In the presence of the $D^{\star}$ formula (52) and coskew symmetry (53), the coassociator formula (54) is equivalent to the cocommutator formula (16).

Proof. Assuming that the coassociator formula (54) holds, we multiply it by $e^{y D^{\star}}$ after replacing $y$ by $-y$ to obtain

$$
\begin{aligned}
& (\lambda(z) \otimes \mathrm{Id}) \wedge(-y) e^{y D^{\star}} \\
& =(\operatorname{Id} \otimes \wedge(-y)) \wedge(z-y) e^{y D^{\star}} \\
& \quad-\operatorname{Res}_{x} \delta(y+x, z)(T \otimes \mathrm{Id}) \\
& \quad \times(\operatorname{Id} \otimes \lambda(x)) \wedge(-y) e^{y D^{\star}}
\end{aligned}
$$

which due to (53) and (57) implies

$$
\begin{aligned}
& (\lambda(z) \otimes \operatorname{Id}) T \wedge(y) \\
& =(\operatorname{Id} \otimes T \wedge(y)) \wedge(z)-\operatorname{Res}_{x} \delta(y+x, z) \\
& \quad \times(T \otimes \operatorname{Id})(\operatorname{Id} \otimes \wedge(x)) T \wedge(y) .
\end{aligned}
$$

Thus, we have that

$$
\begin{aligned}
(\operatorname{Id} \otimes & T \wedge(y)) \wedge(z)-(\wedge(z) \otimes \operatorname{Id}) T \wedge(y) \\
= & \operatorname{Res}_{x} \delta(y+x, z)(T \otimes \operatorname{Id}) \\
& \times(\operatorname{Id} \otimes \wedge(x)) T \wedge(y) .
\end{aligned}
$$

Using that $\delta(y+x, z)=\delta(z-x, y)$ and applying $(\operatorname{Id} \otimes T)$ to (61), we obtain

$$
\begin{aligned}
&(\mathrm{Id}\otimes T)(\mathrm{Id} \otimes T \wedge(y)) \wedge(z)-(\mathrm{Id} \otimes T)(\lambda(z) \otimes \mathrm{Id}) T \wedge(y) \\
& \quad=\operatorname{Res}_{x} \delta(z-x, y)(\mathrm{Id} \otimes T)(T \otimes \mathrm{Id})(\mathrm{Id} \otimes \lambda(x)) T \wedge(y) .
\end{aligned}
$$

Using that

$$
(\mathrm{Id} \otimes T)(\operatorname{Id} \otimes T \wedge(y)) \wedge(z)=(\operatorname{Id} \otimes \wedge(y)) \wedge(z),
$$$$
(\mathrm{Id} \otimes T)(\lambda(z) \otimes \mathrm{Id}) T \wedge(y)=(T \otimes \operatorname{Id})(\mathrm{Id} \otimes \wedge(z)) \wedge(y),
$$$$
(\mathrm{Id} \otimes T)(T \otimes \mathrm{Id})(\mathrm{Id} \otimes \lambda(x)) T \wedge(y)=(\lambda(x) \otimes \mathrm{Id}) \wedge(y),
$$

we have that (62) is equivalent to

$$
\begin{aligned}
(\mathrm{Id} & \otimes \wedge(y)) \wedge(z)-(T \otimes \mathrm{Id})(\mathrm{Id} \otimes \lambda(z)) \wedge(y) \\
& =\operatorname{Res}_{x} \delta(z-x, y)(\lambda(x) \otimes \mathrm{Id}) \wedge(y),
\end{aligned}
$$

which is the cocommutator formula. Using the same argument in reverse order, it is clear that, in the presence of (52) and (53), the cocommutator and the coassociator formulas are equivalent.

Now, we can finish the proof of Theorem 11. By Remarks 3, it remains to show that Definition 10 implies the co-Jacobi identity (15). On the one hand, the coefficient version of the coassociator formula (54) implies the co-Borcherds identity for all $q, r \in \mathbb{Z}$ with fixed $p_{0}=0$. On the other hand, the cocommutator formula, proven to hold under the axioms of Definition 10 in Lemma 13, implies the co-Borcherds identity for all $p, q \in \mathbb{Z}$ with fixed $r_{0}=0$. Therefore, using Theorem 9, we have that the axioms of Definition 10 imply the co-Borcherds identity for all $p, q, r \in \mathbb{Z}$. Now, it is clear that the co-Jacobi identity holds due to the equivalence between Definitions 4 and 1.

Next, we will introduce a definition mainly based on the cocommutator formula. In order to prove its equivalence with the definitions previously introduced, we will show that the coskew symmetry can be deduced from the cocommutator formula in the presence of certain axioms of $D^{\star}$ and $c$.

Definition 14. A vertex coalgebra consists of a $\mathbb{C}$-vector space $V$, together with linear maps

$$
\begin{aligned}
& \wedge(x): V \longrightarrow(V \otimes V)\left(\left(x^{-1}\right)\right), \\
& c: V \longrightarrow \mathbb{C}, \\
& D^{\star}: V \longrightarrow V
\end{aligned}
$$

satisfying the following axioms.

(i) Left counit:

$$
(c \otimes \mathrm{Id}) \wedge(x) v=v, \quad \forall v \in V .
$$

(ii) Cocreation:

$$
e^{x D^{\star}}=(\operatorname{Id} \otimes c) \wedge(x) .
$$


(iii) $D^{\star}$ formula:

$$
\frac{d}{d x} \wedge(x)=\left(D^{\star} \otimes \mathrm{Id}\right) \wedge(x) .
$$

(iv) $D^{\star}$-bracket formula:

$$
\frac{d}{d x} \wedge(x)=\wedge(x) D^{\star}-\left(\operatorname{Id} \otimes D^{\star}\right) \wedge(x) .
$$

(v) Cocommutator formula:

$$
\begin{aligned}
\operatorname{Res}_{z} \delta(x-z, y)(\lambda(z) \otimes I d) \wedge(y) \\
\quad=(\operatorname{Id} \otimes \wedge(y)) \wedge(x)-(T \otimes I d)(\operatorname{Id} \otimes \wedge(x)) \wedge(y) .
\end{aligned}
$$

Our goal is to prove the following statement.

\section{Theorem 15. Definition 14 is equivalent to Definition 10.}

Due to Proposition 12 and Lemma 13, it is clear that Definition 10 implies the axioms of Definition 14. In light of Lemma 13, in order to prove the other implication it is enough to show that coskew symmetry (53) follows from the axioms of Definition 14. We will use the cocommutator formula (70) and the coefficient cocommutator formula (39) interchangeably because, as we mentioned before, (39) is merely the coefficient version of (70).

First, we will need the following result, which is a dual version of the proposition presented in Section 3.2.9 [12] for the case of vertex algebras.

Lemma 16. In the presence of (68) and (69), the coefficient coskew symmetry (41) for indices $r \geq-1$ imply the coefficient coskew symmetry for every $r \in \mathbb{Z}$.

Proof. Note that it is enough to show that for fixed $r \neq 0$, the coefficient coskew symmetry for the index $r$ implies the coefficient coskew symmetry for the index $r-1$. We will denote the coefficient coskew symmetry for fixed $r \in \mathbb{Z}$ by $\operatorname{coskew}(r)$; that is,

$$
\operatorname{coskew}(r): T \Delta_{r}=\sum_{i \geq 0}(-1)^{r+1+i} \Delta_{r+i} D^{\star(i)} .
$$

We assume $\operatorname{coskew}(r)$ for $r \neq 0$ and we want to prove $\operatorname{coskew}(r-1)$. First, we write

$$
\begin{aligned}
r & \cdot\left(\sum_{i \geq 0}(-1)^{r+i} \Delta_{r-1+i} D^{\star(i)}\right) \\
& =\operatorname{Res}_{x}\left(\frac{d}{d x} x^{r}\right) \wedge(-x) e^{x D^{\star}} \\
& =-\operatorname{Res}_{x} x^{r}\left(\frac{d}{d x}\left(\wedge(-x) e^{x D^{\star}}\right)\right) \\
& =-\operatorname{Res}_{x} x^{r}\left(-\left(D^{\star} \otimes \mathrm{Id}\right) \wedge(-x) e^{x D^{\star}}+\lambda(-x) e^{x D^{\star}} D^{\star}\right)
\end{aligned}
$$

due to (68). Using coskew $(r)$ we can rewrite the right hand side of (72) to obtain

$$
\begin{aligned}
r & \cdot\left(\sum_{i \geq 0}(-1)^{r+i} \Delta_{r-1+i} D^{\star(i)}\right) \\
& =-\operatorname{Res}_{x} x^{r}\left(-\left(D^{\star} \otimes \mathrm{Id}\right) T \wedge(x)+T \wedge(x) D^{\star}\right) .
\end{aligned}
$$

Now, we apply $T$ to (69) and use that $T\left(\operatorname{Id} \otimes D^{\star} \lambda(x)=\left(D^{\star} \otimes\right.\right.$ Id) $T \wedge(x)$ to get

$$
\begin{aligned}
r & \left(\sum_{i \geq 0}(-1)^{r+i} \Delta_{r-1+i} D^{\star(i)}\right) \\
& =-\operatorname{Res}_{x} x^{r}\left(\frac{d}{d x} T \wedge(x)\right)=r T \Delta_{r-1} .
\end{aligned}
$$

Dividing by $r$, which we have assumed to be nonzero, we obtain from (74) coskew $(r-1)$, finishing the proof of the lemma.

For the proof of Theorem 15 we will also need the following result.

Proposition 17. Let $\left(V \wedge(x), c, D^{\star}\right)$ be a vertex coalgebra as in Definition 14. Then, the coefficient coskew symmetry, denoted coskew $(r)$ in (71), holds for $r \geq-1$.

Proof. First, let us recall that the cocommutator formula (70) is equivalent to the coefficient cocommutator formula (39) for every $p, q \in \mathbb{Z}$. Second, note that the coefficient cocommutator formula for fixed indices $p, q \in \mathbb{Z}$ is exactly the co-Borcherds identity (34) for indices $p, q \in \mathbb{Z}$ and $r=0$. Now, due to Proposition 8 , it is clear that the co-Borcherds identity for every $p, q \in \mathbb{Z}$ and $r=0$ implies the co-Borcherds identity for every $p, q \in \mathbb{Z}$ and $r \geq 0$. In particular, the cocommutator formula implies the co-Borcherds identity for $p=0, q=-1$, and $r \geq 0$ :

$$
\begin{aligned}
\left(\Delta_{r} \otimes \mathrm{Id}\right) \Delta_{-1} & \\
=\sum_{i \geq 0}(-1)^{i}\left(\begin{array}{c}
r \\
i
\end{array}\right) & {\left[\left(\mathrm{Id} \otimes \Delta_{-1+i}\right) \Delta_{r-i}\right.} \\
& \left.\quad-(-1)^{r}(T \otimes \operatorname{Id})\left(\operatorname{Id} \otimes \Delta_{i}\right) \Delta_{-1+r-i}\right] .
\end{aligned}
$$

Applying $(T \otimes I d)$ to $(75)$ and reordering the terms, we obtain

$$
\begin{aligned}
& \left(T \Delta_{r} \otimes \mathrm{Id}\right) \Delta_{-1} \\
& =\sum_{i \geq 0}(-1)^{i}\left(\begin{array}{c}
r \\
i
\end{array}\right)(-1)^{r+1}\left[\left(\mathrm{Id} \otimes \Delta_{i}\right) \Delta_{-1+r-i}-(-1)^{r}\right. \\
& \left.\times(T \otimes \mathrm{Id})\left(\mathrm{Id} \otimes \Delta_{-1+i}\right) \Delta_{r-i}\right] .
\end{aligned}
$$

As we mentioned before, the co-Borcherds identity (34) holds for $p, q \in \mathbb{Z}$ and $r \geq 0$. Therefore, evaluating $p=-1, q=0$, 
and $r \geq 0$ in (34), the right hand side of (76) can be rewritten to get

$$
\left(T \Delta_{r} \otimes \mathrm{Id}\right) \Delta_{-1}=(-1)^{r+1} \sum_{i \geq 0}\left(\begin{array}{c}
-1 \\
i
\end{array}\right)\left(\Delta_{r+i} \otimes \mathrm{Id}\right) \Delta_{-1-i} .
$$

Next, using that $\left(\begin{array}{c}-1 \\ i\end{array}\right)=(-1)^{i}$ for $i \geq 0$, we apply $(\operatorname{Id} \otimes \operatorname{Id} \otimes c)$ to (77) and reorder the terms to obtain

$$
\left(T \Delta_{r} \otimes \mathrm{Id}\right)(\mathrm{Id} \otimes c) \Delta_{-1}=\sum_{i \geq 0}(-1)^{r+1+i} \Delta_{r+i}(\mathrm{Id} \otimes c) \Delta_{-1-i} .
$$

Using Remarks 2 and 3(b), together with (67) and (68), it is clear that for $i \geq 0$,

$$
D^{\star(i)}=(\operatorname{Id} \otimes \mathcal{C}) \Delta_{-1-i} .
$$

Replacing (79) in (78) we have

$$
\left(T \Delta_{r} \otimes \mathrm{Id}\right)(\mathrm{Id} \otimes c) \Delta_{-1}=\sum_{i \geq 0}(-1)^{r+1+i} \Delta_{r+i} D^{\star(i)},
$$

which due to (67) implies

$$
T \Delta_{r}=\sum_{i \geq 0}(-1)^{r+1+i} \Delta_{r+i} D^{\star(i)} .
$$

Therefore, we have proved that the cocommutator formula (70) implies coskew $(r)$ for $r \geq 0$, in the presence of (67) and (68).

In order to finish the proof, we need to show that coskew $(-1)$ also follows from the axioms of Definition 14. To prove that, we use the coefficient cocommutator formula (39) for $p=-1, q=-1$ :

$$
\begin{aligned}
& \sum_{i \geq 0}\left(\begin{array}{c}
-1 \\
i
\end{array}\right)\left(\Delta_{i} \otimes \mathrm{Id}\right) \Delta_{-2-i} \\
& \quad=\left(\operatorname{Id} \otimes \Delta_{-1}\right) \Delta_{-1}-(T \otimes \mathrm{Id})\left(\operatorname{Id} \otimes \Delta_{-1}\right) \Delta_{-1} .
\end{aligned}
$$

Applying (Id $\otimes \mathrm{Id} \otimes c)$ to (82) and using (79) together with $\left(\begin{array}{c}-1 \\ i\end{array}\right)=(-1)^{i}$ for $i \geq 0$, we obtain

$$
\begin{aligned}
\sum_{i \geq 0}( & -1)^{i} \Delta_{i} D^{\star(i+1)} \\
= & (\operatorname{Id} \otimes \operatorname{Id} \otimes c)\left(\operatorname{Id} \otimes \Delta_{-1}\right) \Delta_{-1} \\
& \quad-(\operatorname{Id} \otimes \operatorname{Id} \otimes c)(T \otimes I d)\left(I d \otimes \Delta_{-1}\right) \Delta_{-1} .
\end{aligned}
$$

Due to (67), it follows that (83) implies

$$
\sum_{i \geq 0}(-1)^{i} \Delta_{i} D^{\star(i+1)}=\Delta_{-1}-T \Delta_{-1},
$$

which is equivalent to coskew $(-1)$. Therefore, we have seen that under the axioms of Definition 14, the cocommutator formula implies $\operatorname{coskew}(r)$ for every $r \geq-1$.
Now we can complete the proof of Theorem 15. Using Proposition 17 together with Lemma 16 it is clear that Definition 14 implies the coskew symmetry (53). With the coskew symmetry proved, the coassociator formula (54) follows from Lemma 13 together with (67), (68), and (70), finishing the proof of the theorem.

\section{Bakalov-Kac Dual Definition}

In this section we will introduce the last definition, which is based on the work of Bakalov and Kac [9] for the case of vertex algebras. They present a vertex algebra as a sort of deformation of a Poisson vertex algebra, namely, a $\mathbb{C}[D]$ Lie conformal algebra and a left-symmetric $\mathbb{C}[D]$-differential algebra with certain compatibilities. In light of (36), it is clear that the $n$-coproducts $\Delta_{n}$ for $n<0$ can be described using the -1 -coproduct and the map $D^{\star}$. Therefore, the coalgebra structure is fully determined by the coproducts $\Delta_{n}$ for $n \geq-1$. That is the motivation for the definition that we present in this section, where the axioms are reformulated to consider properties regarding the $n$-coproducts only for $n \geq-1$.

Let $\left(V, \wedge(x), c, D^{\star}\right)$ be a vertex coalgebra, where

$$
\wedge(x)=\sum_{n \in \mathbb{Z}} \Delta_{n} x^{-n-1} .
$$

In order to consider the -1 -coproduct and the $n$-coproducts for $n \geq 0$, we define two linear maps:

$$
\begin{aligned}
\Lambda: V & \longrightarrow V \otimes V \\
a & \longmapsto \operatorname{Res}_{x} x^{-1} \wedge(x) a=\Delta_{-1}(a), \\
\Delta_{\lambda}: V & \longrightarrow(V \otimes V)[[\lambda]] \\
a & \longmapsto \operatorname{Res}_{x} e^{x \lambda} \wedge(x) a=\sum_{n \geq 0} \Delta_{n}(a) \frac{\lambda^{n}}{n !} .
\end{aligned}
$$

Remarks 4 . Note that the map $\Delta_{\lambda}$ defined in (87) may contain infinitely many powers of $\lambda$, unlike the case of vertex algebras (cf. Section 4 in [9]), where the $\lambda$-bracket that encodes the $n$-products for $n \geq 0$ is truncated.

We want to prove several properties of the maps defined above that will be axioms in the next definition of vertex coalgebra. First, we need to introduce the $(n$-)coproduct identity:

$$
\begin{aligned}
\left(\Delta_{n} \otimes \mathrm{Id}\right) & \wedge(y) \\
=\operatorname{Res}_{x} & {\left[(x-y)^{n}(\operatorname{Id} \otimes \wedge(y)) \wedge(x)\right.} \\
& \left.\quad-(-y+x)^{n}(T \otimes \operatorname{Id})(\operatorname{Id} \otimes \wedge(x)) \wedge(y)\right] .
\end{aligned}
$$

We have the following result.

Proposition 18. The coassociator formula (54) holds if and only if the (n-)coproduct identity (88) holds for all $n \in \mathbb{Z}$.

Proof. Using that $(\operatorname{Id} \lambda(y)) \wedge(z+y)=\operatorname{Res}_{x} \delta(x-y, z)(\operatorname{Id} \otimes$ $\lambda(y)) \lambda(x)$ and taking the coefficient of $z^{-n-1}$ for each $n \in \mathbb{Z}$ in (54), the equivalence is clear. 

later.

Now we can prove the list of properties that we will need

Proposition 19. Let $\left(V, \lambda(x), c, D^{\star}\right)$ be a vertex coalgebra and let $\Lambda, \Delta_{\lambda}$ be the maps defined in (86) and (87), respectively. Then, the following conditions are satisfied.

(1) Counit:

$$
(c \otimes \mathrm{Id}) \Lambda=\mathrm{Id}=(\mathrm{Id} \otimes c) \Lambda .
$$

(2) Coderivation with respect to $\Lambda$ :

$$
\Lambda D^{\star}=\left[\left(D^{\star} \otimes \mathrm{Id}\right)+\left(\operatorname{Id} \otimes D^{\star}\right)\right] \Lambda .
$$

(3) Counit holomorphic condition:

$$
(\mathrm{Id} \otimes c) \Delta_{\lambda}=0 .
$$

(4) $D^{\star}$-sesquilinearity:

$$
\begin{gathered}
\left(D^{\star} \otimes \mathrm{Id}\right) \Delta_{\lambda}=\Delta_{\lambda}(-\lambda), \\
\left(\mathrm{Id} \otimes D^{\star}\right) \Delta_{\lambda}=\Delta_{\lambda}\left(\lambda+D^{\star}\right) .
\end{gathered}
$$

(5) Conformal coskew symmetry:

$$
T \Delta_{\lambda}=-\Delta_{-\lambda-D^{*}}
$$

(6) Conformal co-Jacobi formula:

$$
\left(\Delta_{\lambda} \otimes \mathrm{Id}\right) \Delta_{\lambda+\mu}=\left(\operatorname{Id} \otimes \Delta_{\mu}\right) \Delta_{\lambda}-(T \otimes \operatorname{Id})\left(\mathrm{Id} \otimes \Delta_{\lambda}\right) \Delta_{\mu} .
$$

(7) Co-Wick formula:

$$
\begin{aligned}
\left(\Delta_{\lambda} \otimes \mathrm{Id}\right) \Lambda= & (\operatorname{Id} \otimes \Lambda) \Delta_{\lambda}-(T \otimes \mathrm{Id})\left(\operatorname{Id} \otimes \Delta_{\lambda}\right) \Lambda \\
& -\int_{0}^{\lambda}\left(\Delta_{\lambda} \otimes \mathrm{Id}\right) \Delta_{\mu} d \mu .
\end{aligned}
$$

(8) Cocompatibility:

$$
\Lambda=T \Lambda+\int_{-D^{\star}}^{0} \Delta_{\lambda} d \lambda
$$

(9) Coleft symmetry:

$$
\begin{aligned}
& (\operatorname{Id} \otimes \Lambda) \Lambda-(T \otimes \operatorname{Id})(\operatorname{Id} \otimes \Lambda) \Lambda \\
& \quad=(\Lambda \otimes \operatorname{Id}) \Lambda-(T \otimes \operatorname{Id})(\Lambda \otimes \operatorname{Id}) \Lambda .
\end{aligned}
$$

Remarks 5. (a) The expression $\Delta_{-\lambda-D^{\star}}$ is interpreted as follows:

$$
\Delta_{-\lambda-D^{\star}}(v)=\sum_{n \geq 0} \frac{\Delta_{n}}{n !}\left(-\lambda-D^{\star}\right)^{n}(v),
$$

with the powers of $-\lambda-D^{\star}$ on the right, unlike the case of $\mathbb{C}[D]$-Lie conformal algebras in [9]. Moreover, using that
$D^{\star}$ is locally nilpotent (see Proposition 5(3)), it is clear that $\Delta_{-\lambda-D^{\star}}$ makes sense in $\operatorname{Hom}(V,(V \otimes V)[[\lambda]])$.

(b) Note that the expression $\left(\Delta_{\lambda} \otimes \mathrm{Id}\right) \Delta_{\lambda+\mu}$ is well defined in $\operatorname{Hom}(V,(V \otimes V)[[\lambda, \mu]])$.

(c) The integral notation in (7) and (8) is interpreted as follows:

$$
\begin{aligned}
& \int_{0}^{\lambda}\left(\Delta_{\lambda} \otimes \mathrm{Id}\right) \Delta_{\mu} d \mu \\
& \quad=\sum_{n, m \geq 0}\left(\Delta_{n} \otimes \mathrm{Id}\right) \Delta_{m} \frac{\lambda^{n+m+1}}{n !(m+1) !}, \\
& \int_{-D^{\star}}^{0} \Delta_{\lambda} d \lambda=-\sum_{n \geq 0} \Delta_{n}\left(-D^{\star}\right)^{(n+1)},
\end{aligned}
$$

unlike the case of Lie conformal algebras, where the parameter and its evaluation on $D$ are always placed on the left. Indeed, from now on expansions inside definite integrals will be interpreted with all the powers of the parameters $\lambda$ and $\mu$ placed on the right.

(d) The names of properties (1)-(9) in Proposition 19 are motivated by the Definition of vertex algebra presented in Section 6 [9]. Formulas (1), (2), and (9) are the dual version of the axioms of a left symmetric $\mathbb{C}[D]$-differential algebra with unit (cf. Section 4 [9]). Formula (3) is related to the fact that $(\operatorname{Id} \otimes c) \wedge(x)$ is holomorphic. On the other hand, (4), (5), and (6) are dual to the axioms of a $\mathbb{C}[D]$-Lie conformal algebra. Finally, (7) and (8) relate the two structures in a dual version of the Wick formula and the compatibility described in Theorem 6.9 [9].

Proof of Proposition 19. (1) The proof follows from (12) and (13).

(2) It is clear if we take the coefficient of $x^{0}$ in (22) and

(3) In light of (13), it is clear that $(\operatorname{Id} \otimes c) \Delta_{\lambda}=$ $\operatorname{Res}_{x} e^{\lambda x}(\operatorname{Id} \otimes c) \wedge(x)=0$.

(4) Using (22), we have

$$
\begin{aligned}
\left(D^{\star} \otimes \mathrm{Id}\right) \Delta_{\lambda} & =\operatorname{Res}_{x} e^{\lambda x}\left(D^{\star} \otimes \mathrm{Id}\right) \wedge(x) \\
& =-\operatorname{Res}_{x}\left(\frac{d}{d x} e^{\lambda x}\right) \wedge(x)=\Delta_{\lambda}(-\lambda) .
\end{aligned}
$$

Using (26) and part (4) proved above we have

$$
\begin{aligned}
\left(\operatorname{Id} \otimes D^{\star}\right) \Delta_{\lambda} & =\operatorname{Res}_{x} e^{\lambda x}\left(-\frac{d}{d x} \lambda(x)+\wedge(x) D^{\star}\right) \\
& =\Delta_{\lambda}\left(\lambda+D^{\star}\right)
\end{aligned}
$$

(5) Using (25) we have

$$
T \Delta_{\lambda}=\operatorname{Res}_{x} e^{\lambda x} T \wedge(x)=\operatorname{Res}_{x} \wedge(-x) e^{x\left(\lambda+D^{\star}\right)}=-\Delta_{-\lambda-D^{\star}} .
$$


(6) Note that for $n \geq 0$ the (n-)coproduct formula (88) can be rewritten as follows:

$$
\begin{aligned}
\left(\Delta_{n} \otimes \mathrm{Id}\right) & \wedge(y) \\
=\operatorname{Res}_{x}(x-y)^{n}((\operatorname{Id} \otimes & \wedge(y)) \wedge(x) \\
& \quad-(T \otimes \operatorname{Id})(\operatorname{Id} \otimes \lambda(x)) \wedge(y)) .
\end{aligned}
$$

Changing the variables $x$ for $z$ and $y$ for $x$ in (103) we obtain that the collection of ( $n$-) coproduct identities (88) for $n \geq 0$ is equivalent to

$$
\begin{aligned}
&\left(\Delta_{\lambda} \otimes \mathrm{Id}\right) \wedge(x)= \sum_{n \geq 0}\left(\Delta_{n} \otimes \mathrm{Id}\right) \wedge(x) \frac{\lambda^{n}}{n !} \\
&= \sum_{n \geq 0} \operatorname{Res}_{z}(z-x)^{n} \\
& \times((\operatorname{Id} \otimes \wedge(x)) \wedge(z)-(T \otimes \mathrm{Id}) \\
&\times(\operatorname{Id} \otimes \wedge(z)) \wedge(x)) \frac{\lambda^{n}}{n !} \\
&= \operatorname{Res}_{z} e^{\lambda(z-x)} \\
& \quad \times((\operatorname{Id} \otimes \wedge(x)) \wedge(z)-(T \otimes \operatorname{Id}) \\
&\quad \times(\operatorname{Id} \otimes \lambda(z)) \wedge(x)) \\
&=e^{-\lambda x}\left((\operatorname{Id} \otimes \lambda(x)) \Delta_{\lambda}\right. \\
&\left.\quad-(T \otimes \operatorname{Id})\left(\operatorname{Id} \otimes \Delta_{\lambda}\right) \wedge(x)\right) .
\end{aligned}
$$

Multiplying (104) by $e^{(\lambda+\mu) x}$ and taking $\operatorname{Res}_{x}$ we obtain

$$
\left(\Delta_{\lambda} \otimes \mathrm{Id}\right) \Delta_{\lambda+\mu}=\left(\operatorname{Id} \otimes \Delta_{\mu}\right) \Delta_{\lambda}-(T \otimes \mathrm{Id})\left(\mathrm{Id} \otimes \Delta_{\lambda}\right) \Delta_{\mu} .
$$

(7) Multiplying (104) by $x^{-1}$, using that $e^{-\lambda x} x^{-1}=x^{-1}+$ $\int_{0}^{-\lambda} e^{\mu x} d \mu$ and taking $\operatorname{Res}_{x}$ we have

$$
\begin{aligned}
\left(\Delta_{\lambda} \otimes \mathrm{Id}\right) \Lambda= & (\mathrm{Id} \otimes \Lambda) \Delta_{\lambda}-(T \otimes \operatorname{Id})\left(\operatorname{Id} \otimes \Delta_{\lambda}\right) \Lambda \\
& +\int_{0}^{-\lambda}\left(\left(\operatorname{Id} \otimes \Delta_{\mu}\right) \Delta_{\lambda}\right. \\
& \left.\quad-(T \otimes \operatorname{Id})\left(\operatorname{Id} \otimes \Delta_{\lambda}\right) \Delta_{\mu}\right) d \mu .
\end{aligned}
$$

Using the conformal co-Jacobi formula (6) and making a change of variables we can rewrite (106) as follows:

$$
\begin{aligned}
\left(\Delta_{\lambda} \otimes \mathrm{Id}\right) \Lambda= & (\mathrm{Id} \otimes \Lambda) \Delta_{\lambda}-(T \otimes \mathrm{Id})\left(\operatorname{Id} \otimes \Delta_{\lambda}\right) \Lambda \\
& -\int_{0}^{\lambda}\left(\Delta_{\lambda} \otimes \mathrm{Id}\right) \Delta_{\mu} d \mu .
\end{aligned}
$$

(8) Using that $x^{-1} e^{x D^{\star}}=x^{-1}-\int_{0}^{-D^{\star}} e^{-\lambda x} d \lambda$ and coskew symmetry (25) we have

$$
\begin{aligned}
\Lambda & =\operatorname{Res}_{x} x^{-1} \wedge(x) \\
& =\operatorname{Res}_{x} T \wedge(-x) x^{-1} e^{x D^{\star}}
\end{aligned}
$$

$$
\begin{aligned}
& =\operatorname{Res}_{x} T \lambda(-x) x^{-1} \\
& \quad-\int_{0}^{-D^{\star}} \operatorname{Res}_{x} T \lambda(-x) e^{-\lambda x} d \lambda \\
& =T \Lambda+\int_{0}^{-D^{\star}} T \Delta_{\lambda} d \lambda .
\end{aligned}
$$

Using conformal coskew symmetry proved in (5) and making a change of variables we can rewrite (108) as follows:

$$
\Lambda=T \Lambda+\int_{-D^{\star}}^{0} \Delta_{\lambda} d \lambda
$$

(9) In order to prove coleft symmetry we will follow the argument developed by Bakalov and Kac for the case of vertex algebras in [9]. We start by proving the following formula:

$$
\begin{aligned}
& (\Lambda \otimes \mathrm{Id}) \Lambda-(\operatorname{Id} \otimes \Lambda) \Lambda \\
& =\left(\int_{0}^{D^{\star} \otimes \mathrm{Id}}\left(\operatorname{Id} \otimes \Delta_{\lambda}\right) d \lambda\right) \Lambda \\
& \quad+(T \otimes \operatorname{Id})\left(\int_{0}^{D^{\star} \otimes \mathrm{Id}}\left(\operatorname{Id} \otimes \Delta_{\lambda}\right) d \lambda\right) \Lambda,
\end{aligned}
$$

where the integral notation is interpreted as follows: first we expand the $\lambda$ coproduct and put the powers of $\lambda$ on the right inside the integral and then we take the definite integral by the usual rules inside the parenthesis. Namely,

$$
\begin{aligned}
& \left(\int_{0}^{D^{\star} \otimes \mathrm{Id}}\left(\operatorname{Id} \otimes \Delta_{\lambda}\right) d \lambda\right) \Lambda \\
& \quad=\left(\int_{0}^{D^{\star} \otimes \mathrm{Id}} \sum_{n \geq 0}\left(\operatorname{Id} \otimes \Delta_{n}\right) \frac{\lambda^{n}}{n !} d \lambda\right) \Lambda \\
& \quad=\sum_{n \geq 0}\left(\left(D^{\star}\right)^{(n+1)} \otimes \Delta_{n}\right) \Lambda .
\end{aligned}
$$

In order to prove (110) we will use the (-1)-coproduct formula proved in Proposition 18:

$$
\begin{aligned}
\left(\Delta_{-1} \otimes \mathrm{Id}\right) & \wedge(y) \\
=\operatorname{Res}_{x}( & (x-y)^{-1}(\operatorname{Id} \otimes \wedge(y)) \wedge(x)-(-y+x)^{-1} \\
& \times(T \otimes \operatorname{Id})(\operatorname{Id} \otimes \wedge(x)) \wedge(y)) .
\end{aligned}
$$


We multiply (112) by $y^{-1}$, take $\operatorname{Res}_{y}$, and use that $\left(\begin{array}{c}-1 \\ k\end{array}\right)=(-1)^{k}$ for $k \geq 0$ to obtain

$$
\begin{aligned}
& (\Lambda \otimes \mathrm{Id}) \Lambda \\
& =\operatorname{Res}_{y} y^{-1}\left(\operatorname { R e s } _ { x } \left((x-y)^{-1}(\operatorname{Id} \otimes \wedge(y)) \wedge(x)\right.\right. \\
& -(-y+x)^{-1}(T \otimes \mathrm{Id}) \\
& \times(\operatorname{Id} \otimes \wedge(x)) \wedge(y))) \\
& =\operatorname{Res}_{y} y^{-1}\left(\sum _ { k \geq 0 } ( - 1 ) ^ { k } \left((-y)^{k}(\operatorname{Id} \otimes \wedge(y)) \Delta_{-k-1}\right.\right. \\
& -(-y)^{-k-1}(T \otimes \mathrm{Id}) \\
& \left.\left.\times\left(\operatorname{Id} \otimes \Delta_{k}\right) \wedge(y)\right)\right) \\
& =\operatorname{Res}_{y} y^{-1}\left(\sum _ { n \in \mathbb { Z } } \sum _ { k \geq 0 } \left(y^{-n-1+k}\left(\operatorname{Id} \otimes \Delta_{n}\right) \Delta_{-k-1}\right.\right. \\
& \left.\left.+y^{-n-2-k}(T \otimes \operatorname{Id})\left(\operatorname{Id} \otimes \Delta_{k}\right) \Delta_{n}\right)\right) \\
& =\sum_{k \geq 0}\left(\left(\operatorname{Id} \otimes \Delta_{k-1}\right) \Delta_{-k-1}\right. \\
& \left.+(T \otimes \operatorname{Id})\left(\operatorname{Id} \otimes \Delta_{k}\right) \Delta_{-k-2}\right) \\
& =\left(\operatorname{Id} \otimes \Delta_{-1}\right) \Delta_{-1} \\
& +\sum_{k \geq 1}\left(\left(\mathrm{Id} \otimes \Delta_{k-1}\right) \Delta_{-k-1}\right. \\
& \left.+(T \otimes \operatorname{Id})\left(\operatorname{Id} \otimes \Delta_{k-1}\right) \Delta_{-k-1}\right) .
\end{aligned}
$$

Using that $\left(\left(D^{\star}\right)^{(j)} \otimes \mathrm{Id}\right) \Delta_{-1}=\Delta_{-1-j}$ for $j \geq 0$, which follows from (36), we can rewrite the right hand side of (113) to get

$$
\begin{aligned}
(\Lambda \otimes \mathrm{Id}) \Lambda=(\operatorname{Id} \otimes \Lambda) & \\
+\sum_{k \geq 1}( & \left(\mathrm{Id} \otimes \Delta_{k-1}\right)\left(\left(D^{\star}\right)^{(k)} \otimes \mathrm{Id}\right) \\
& +(T \otimes \mathrm{Id})\left(\mathrm{Id} \otimes \Delta_{k-1}\right) \\
& \left.\times\left(\left(D^{\star}\right)^{(k)} \otimes \mathrm{Id}\right)\right) \Lambda .
\end{aligned}
$$

Using (111) it is easy to check that the right hand side of (114) equals

$$
\begin{aligned}
& (\operatorname{Id} \otimes \Lambda) \Lambda+\left(\int_{0}^{D^{*} \otimes \operatorname{Id}}\left(\operatorname{Id} \otimes \Delta_{\lambda}\right) d \lambda\right) \Lambda \\
& +(T \otimes \operatorname{Id})\left(\int_{0}^{D^{*} \otimes I \mathrm{I}}\left(\operatorname{Id} \otimes \Delta_{\lambda}\right) d \lambda\right) \Lambda,
\end{aligned}
$$

finishing the proof of (110).
In order to complete the proof of (9), we note that the right hand side of (110) is invariant under the operator $(T \otimes$ Id). Therefore, its left hand side is invariant under $(T \otimes I d)$ as well. This clearly implies coleft symmetry (97), finishing the proof of Proposition 19.

Let us suppose that we start with a $\mathbb{C}$-vector space $V$ and four linear maps:

$$
\begin{aligned}
c: V \longrightarrow \mathbb{C}, \\
D^{\star}: V \longrightarrow V, \\
\Lambda: V \longrightarrow V \otimes V, \\
\Delta_{\lambda}: V \longrightarrow(V \otimes V)[[\lambda]],
\end{aligned}
$$

such that $D^{\star}$ is locally nilpotent and properties (1)-(9) of Proposition 19 hold. Then, we define a new linear map

$$
\begin{aligned}
\curlywedge(x): & V \longrightarrow(V \otimes V)\left[\left[x, x^{-1}\right]\right] \\
& v \longmapsto \Delta_{-(d / d x)}(v)\left(x^{-1}\right)+\left(e^{x D^{*}} \otimes \mathrm{Id}\right) \Lambda(v),
\end{aligned}
$$

where the expression $\Delta_{-(d / d x)}(v)\left(x^{-1}\right)$ is interpreted as $\Delta_{\lambda}(v)$ evaluated in $\lambda=-d / d x$ acting on $x^{-1}$. Namely, if we write $\Delta_{\lambda}(v)=\sum_{n \geq 0} \Delta_{n}(v)\left(\lambda^{n} / n !\right)$, then

$$
\begin{aligned}
\Delta_{-(d / d x)}(v)\left(x^{-1}\right) & =\sum_{n \geq 0} \Delta_{n}(v)\left(-\frac{d}{d x}\right)^{(n)}\left(x^{-1}\right) \\
& =\sum_{n \geq 0} \Delta_{n}(v) x^{-n-1} .
\end{aligned}
$$

We want to prove that $\left(V, \lambda(x), c, D^{\star}\right)$ with $\lambda(x)$ defined in (117) is a vertex coalgebra. Indeed, we will show that providing a $\mathbb{C}$-vector space with the structure described above is equivalent to providing $V$ with a coalgebra structure.

Remarks 6. (a) If we consider the expansion $\lambda(x)=$ $\sum_{n \in \mathbb{Z}} \Delta_{n} x^{-n-1}$ for the map $\lambda(x)$ defined in (117), then we are defining $\Delta_{-n-1}:=\left(\left(D^{\star}\right)^{(n)} \otimes \mathrm{Id}\right) \Lambda$ for $n \geq 0$.

(b) In this context, we can recover the maps $\Lambda$ and $\Delta_{\lambda}$ using the formulas $\Lambda=\operatorname{Res}_{x} x^{-1} \lambda(x), \Delta_{\lambda}=\operatorname{Res}_{x} e^{\lambda x} \lambda(x)$ with $\lambda(x)$ defined as in (117).

(c) If we have a vertex coalgebra $\left(V, \lambda(x), c, D^{\star}\right)$ and define maps $\Lambda$ and $\Delta_{\lambda}$ as we did in (86), (87), then due to (36) we can reobtain $\lambda(x)$ as in (117).

We introduce the last definition.

Definition 20. A vertex coalgebra consists of a $\mathbb{C}$-vector space $V$ together with linear maps

$$
\begin{aligned}
& c: V \longrightarrow \mathbb{C}, \\
& D^{\star}: V \longrightarrow V, \\
& \Lambda: V \longrightarrow V \otimes V, \\
& \Delta_{\lambda}: V \longrightarrow(V \otimes V)[[\lambda]],
\end{aligned}
$$


such that $D^{\star}$ is locally nilpotent and conditions (1)-(9) of Proposition 19 hold.

As we mentioned before, we want to prove the following result.

\section{Theorem 21. Definition 20 is equivalent to Definition 10.}

In light of the equivalence between Definitions 10 and 1, Propositions 5(3) and 19 imply that the axioms of Definition 20 are satisfied under the conditions of Definition 10 if we define $\Lambda$ and $\Delta_{\lambda}$ as in (86)-(87).

In order to prove the other implication let us first define $\lambda(x)$ as in (117). Using that $D^{\star}$ is locally nilpotent, it is clear that the image of $\lambda(x)$ actually lies in $(V \otimes V)\left(\left(x^{-1}\right)\right)$. Second, note that using (89) and (90) it is clear that $c D^{\star}=0$. Indeed, (89) and (90) imply

$$
\begin{aligned}
c D^{\star}= & c(c \otimes \mathrm{Id}) \Lambda D^{\star}=(c \otimes c) \Lambda D^{\star} \\
= & (c \otimes \mathrm{Id})(\mathrm{Id} \otimes c) \Lambda D^{\star} \\
= & (c \otimes \mathrm{Id})(\mathrm{Id} \otimes c)\left(D^{\star} \otimes \mathrm{Id}\right) \Lambda \\
& +(c \otimes \mathrm{Id})(\mathrm{Id} \otimes c)\left(\mathrm{Id} \otimes D^{\star}\right) \Lambda \\
= & (c \otimes \mathrm{Id})\left(D^{\star} \otimes \mathrm{Id}\right)(\mathrm{Id} \otimes c) \Lambda \\
& +(\mathrm{Id} \otimes c)\left(\mathrm{Id} \otimes D^{\star}\right)(c \otimes \mathrm{Id}) \Lambda \\
= & c D^{\star}+c D^{\star} .
\end{aligned}
$$

Using that $c D^{\star}=0$ together with (91) and (92), we have $(c \otimes \mathrm{Id}) \Delta_{\lambda}=0=(\mathrm{Id} \otimes c) \Delta_{\lambda}$. The fact that $c D^{\star}=0$ together with $(c \otimes \mathrm{Id}) \Delta_{\lambda}=0$ and (89) implies (50). On the other hand, (91) and (89) imply (51). Next, note that (52) follows from (92). Therefore, in order to finish the proof we need to show that coskew symmetry (53) and the coassociator formula (54) follow from Definition 20.

We start with the proof of coskew symmetry. Note that (90) and (92), together with (52), proven to hold above, imply that $\lambda(x)$ satisfies the $D^{\star}$-bracket formula

$$
\wedge(x) D^{\star}=\left(\operatorname{Id} \otimes D^{\star}\right) \wedge(x)+\frac{d}{d x} \wedge(x) .
$$

If we define the linear map

$$
\begin{gathered}
\wedge^{o p}(x): V \longrightarrow(V \otimes V)\left(\left(x^{-1}\right)\right) \\
v \longrightarrow T \wedge(-x) e^{x D^{\star}},
\end{gathered}
$$

it follows that $\wedge^{o p}(x)$ also satisfies

$$
\frac{d}{d x} \wedge^{o p}(x)=\left(D^{\star} \otimes \mathrm{Id}\right) \wedge^{o p}(x) .
$$

Next, we define

$$
\begin{aligned}
\Delta_{\lambda}^{o p} & :=\operatorname{Res}_{x} e^{x \lambda} \lambda^{o p}(x), \\
\Lambda^{o p} & :=\operatorname{Res}_{x} x^{-1} \lambda^{o p}(x) .
\end{aligned}
$$

Using the coefficient version of (123) (cf. proof of Proposition 5(1)) and Remarks 6 it is clear that we can reconstruct

$$
\Lambda^{o p}(x)=\Delta_{-d / d x}^{o p}\left(x^{-1}\right)+\left(e^{x D^{\star}} \otimes \mathrm{Id}\right) \Lambda^{o p} .
$$

Now, conformal coskew symmetry (93) implies

$$
\Delta_{\lambda}^{o p}=\operatorname{Res}_{x} e^{\lambda x} T \lambda(-x) e^{x D^{\star}}=-T \Delta_{-\lambda-D^{\star}}=\Delta_{\lambda},
$$

whereas the fact that $x^{-1} e^{x D^{\star}}=x^{-1}-\int_{0}^{-D^{\star}} e^{-\lambda x} d \lambda$ together with conformal coskew symmetry (93) and cocompatibility (96) implies

$$
\begin{aligned}
\Lambda^{o p} & =\operatorname{Res}_{x} x^{-1} T \lambda(-x) e^{x D^{\star}}=T \Lambda+\int_{0}^{-D^{\star}} T \Delta_{\lambda} d \lambda \\
& =T \Lambda-\int_{0}^{-D^{\star}} \Delta_{-\lambda-D^{\star}} d \lambda=T \Lambda+\int_{-D^{\star}}^{0} \Delta_{\lambda} d \lambda=\Lambda .
\end{aligned}
$$

Therefore, using (126) and (127) in (125) together with the definition of $\lambda(x)$ in (117) it follows that $\lambda^{o p}(x)=\lambda(x)$, which implies coskew symmetry (53).

Finally, we want to prove that the coassociator formula holds. Note that due to Proposition 18(2), it is equivalent to the $(n$-)coproduct identity ( 88$)$ for all $n \in \mathbb{Z}$. If we denote

$$
\begin{aligned}
\operatorname{cop}(n)[\curlywedge(z)]:=\operatorname{Res}_{x}[ & (x-z)^{n}(\operatorname{Id} \otimes \wedge(z)) \wedge(x) \\
- & (-z+x)^{n}(T \otimes \mathrm{Id}) \\
& \times(\operatorname{Id} \otimes \wedge(x)) \wedge(z)]
\end{aligned}
$$

then the $(n$-)coproduct identity is equivalent to

$$
\left(\Delta_{n} \otimes \mathrm{Id}\right) \wedge(z)-\operatorname{cop}(n)[\wedge(z)]=0 .
$$

In order to prove that it holds for every $n \in \mathbb{Z}$ we introduce the following result.

Lemma 22. Let $D^{\star} \in$ EndV be a locally nilpotent map and let

$$
\wedge(x): V \longrightarrow(V \otimes V)\left(\left(x^{-1}\right)\right)
$$

be a linear map satisfying

$$
\begin{gathered}
\wedge(x) D^{\star}=\left(\operatorname{Id} \otimes D^{\star}\right) \wedge(x)+\left(D^{\star} \otimes \mathrm{Id}\right) \wedge(x), \\
\frac{d}{d x} \wedge(x)=\left(D^{\star} \otimes \mathrm{Id}\right) \wedge(x) .
\end{gathered}
$$

Then, $\lambda(x)$ satisfies the ( $n$-)coproduct identity (88) for all $n \in \mathbb{Z}$ if and only if

$$
\operatorname{Res}_{z}\left(\left(\Delta_{n} \otimes \mathrm{Id}\right) \wedge(z)-\operatorname{cop}(n)[\wedge(z)]\right) F=0
$$

for all $n \geq-1$ with $F=z^{-1}$ and $F=e^{\lambda z}$. 
Proof. If we assume that the ( $n$-)coproduct formula (88) holds for every $n \in \mathbb{Z}$, then it is clear that (133) holds for $n \geq-1$. Conversely, if we assume that (133) holds for $n \geq-1$ we will show that it holds with $F$ replaced by $(d / d z) F$. In fact,

$$
\begin{aligned}
& \operatorname{Res}_{z}\left(\left(\Delta_{n} \otimes \mathrm{Id}\right) \wedge(z)-\operatorname{cop}(n)[\wedge(z)]\right)\left(\frac{d}{d z} F\right) \\
& =-\operatorname{Res}_{z}\left(\frac{d}{d z}\left(\left(\Delta_{n} \otimes \mathrm{Id}\right) \wedge(z)-\operatorname{cop}(n)[\wedge(z)]\right)\right) F .
\end{aligned}
$$

Using (131) and the coefficient version of (132) we have that

$$
\begin{aligned}
\frac{d}{d z} & \left(\Delta_{n} \otimes \mathrm{Id}\right) \wedge(z) \\
= & \left(\Delta_{n} \otimes \mathrm{Id}\right)\left(D^{\star} \otimes \mathrm{Id}\right) \wedge(z) \\
= & \left(D^{\star} \otimes \mathrm{Id} \otimes \mathrm{Id}\right)\left(\Delta_{n} \otimes \mathrm{Id}\right) \wedge(z) \\
& +\left(\mathrm{Id} \otimes D^{\star} \otimes \mathrm{Id}\right)\left(\Delta_{n} \otimes \mathrm{Id}\right) \wedge(z),
\end{aligned}
$$

whereas, (132) implies

$$
\begin{aligned}
\frac{d}{d z} \operatorname{cop}(n)[\lambda(z)]= & -n \operatorname{cop}(n-1)[\lambda(z)] \\
& +\left(\operatorname{Id} \otimes D^{\star} \otimes \operatorname{Id}\right) \operatorname{cop}(n)[\lambda(z)] .
\end{aligned}
$$

Using (132) and integration by parts we have

$$
\begin{aligned}
& -n \operatorname{cop}(n-1)[\lambda(z)] \\
& =-\operatorname{Rex}_{x}\left[\left(\frac{d}{d x}(x-z)^{n}\right)(\operatorname{Id} \otimes \lambda(z)) \wedge(x)\right. \\
& -\left(\frac{d}{d x}(-z+x)^{n}\right) \\
& \times(T \otimes \operatorname{Id})(\operatorname{Id} \otimes \wedge(x)) \wedge(z)] \\
& =\left(D^{\star} \otimes \operatorname{Id} \otimes \operatorname{Id}\right) \operatorname{cop}(n)[\wedge(z)] .
\end{aligned}
$$

Replacing (135) and (137) in (134) we obtain

$$
\begin{aligned}
\operatorname{Res}_{z} & \left(\left(\Delta_{n} \otimes \mathrm{Id}\right) \wedge(z)-\operatorname{cop}(n)[\wedge(z)]\right)\left(\frac{d}{d z} F\right) \\
= & -\left(D^{\star} \otimes \mathrm{Id} \otimes \mathrm{Id}\right) \\
& \times \operatorname{Res}_{z}\left[\left(\Delta_{n} \otimes \mathrm{Id}\right) \wedge(z)-\operatorname{cop}(n)[\lambda(z)]\right] F \\
& -\left(\operatorname{Id} \otimes D^{\star} \otimes \mathrm{Id}\right) \\
& \times \operatorname{Res}_{z}\left[\left(\Delta_{n} \otimes \mathrm{Id}\right) \wedge(z)-\operatorname{cop}(n)[\lambda(z)]\right] F \\
= & 0 .
\end{aligned}
$$

Hence, it follows that (133) also holds for all $F=z^{k}, k \leq 0$. Taking coefficients at powers of $\lambda$ for $F=e^{\lambda z}$, we have that (133) also holds for all $F=z^{k}, k \geq 0$. This implies the ( $n$-)coproduct identity (88) for $n \geq-1$. Finally, it follows from the coefficient version of (132) together with (137) that for $n \neq 0$ the $(n$-) coproduct identity implies the $(n-1)$-coproduct identity. Therefore, the $(n$-) coproduct identity holds for all $n \in \mathbb{Z}$.

In light of Lemma 22, in order to finish the proof of Theorem 21 it is enough to prove that (133) holds for all $n \geq-1$ with $F=z^{-1}$ and $F=e^{\lambda z}$. In the proof of Proposition 19(6), we proved that the conformal co-Jacobi formula (94) is equivalent to (133) for all $n \geq 0$ and $F=e^{\lambda z}$. In the proof of (7), we proved that the co-Wick formula (95) together with the conformal co-Jacobi identity (94) implies (133) for all $n \geq 0$ and $F=z^{-1}$. In order to prove the remaining two cases we will need the following result.

Proposition 23. Let $\left(V, \lambda(x), c, D^{\star}\right)$ be a vertex coalgebra as in Definition 20. Then,

(1) $(\Lambda \otimes \mathrm{Id}) \Delta_{\lambda}$

$$
\begin{aligned}
& =\left(e^{D^{\star}(d / d \lambda)} \otimes \Delta_{\lambda}\right) \Lambda \\
& +(T \otimes \mathrm{Id})\left(e^{D^{\star}(d / d \lambda)} \otimes \Delta_{\lambda}\right) \Lambda \\
& \quad+\int_{0}^{\lambda}(T \otimes \mathrm{Id})\left(\operatorname{Id} \otimes \Delta_{\lambda-\mu}\right) \Delta_{\mu} d \mu .
\end{aligned}
$$

(2) $(\Lambda \otimes \operatorname{Id}) \Lambda-(\operatorname{Id} \otimes \Lambda) \Lambda$

$$
\begin{aligned}
& =\left(\int_{0}^{D^{\star} \otimes \operatorname{Id}}\left(\operatorname{Id} \otimes \Delta_{\lambda}\right) d \lambda\right) \Lambda \\
& \quad+(T \otimes \operatorname{Id})\left(\int_{0}^{D^{\star} \otimes \operatorname{Id}}\left(\operatorname{Id} \otimes \Delta_{\lambda}\right) d \lambda\right) \Lambda .
\end{aligned}
$$

Proof. (1) First, using cocompatibility (96) we have

$$
\left(\Delta_{\lambda} \otimes \mathrm{Id}\right) \Lambda=\left(\Delta_{\lambda} \otimes \mathrm{Id}\right) T \Lambda+\int_{-D^{\star}}^{0}\left(\Delta_{\lambda} \otimes \mathrm{Id}\right) \Delta_{\mu} d \mu
$$

which, due to the co-Wick formula (95), implies

$$
\begin{aligned}
(\mathrm{Id} \otimes \Lambda) \Delta_{\lambda}= & \left(\Delta_{\lambda} \otimes \mathrm{Id}\right) T \Lambda+(T \otimes \mathrm{Id})\left(\mathrm{Id} \otimes \Delta_{\lambda}\right) \Lambda \\
& +\int_{-D^{\star}}^{\lambda}\left(\Delta_{\lambda} \otimes \mathrm{Id}\right) \Delta_{\mu} d \mu .
\end{aligned}
$$

Observe that the integral notation in (142) is interpreted as follows: first we expand $\Delta_{\lambda}$ and $\Delta_{\mu}$ inside the integral sign 
putting the powers of $\lambda$ and $\mu$ on the right of the sum. Then, we take the definite integral by the usual rules. Namely,

$$
\begin{aligned}
\int_{-D^{\star}}^{\lambda} & \left(\Delta_{\lambda} \otimes \mathrm{Id}\right) \Delta_{\mu} d \mu \\
= & \int_{-D^{\star}}^{\lambda}\left(\sum_{n, m \geq 0}\left(\Delta_{n} \otimes \mathrm{Id}\right) \Delta_{m} \frac{\lambda^{n}}{n !} \frac{\mu^{m}}{m !}\right) d \mu \\
= & \sum_{n, m \geq 0}\left(\Delta_{n} \otimes \mathrm{Id}\right) \Delta_{m} \frac{\lambda^{n+m+1}}{n !(m+1) !} \\
& -\sum_{m \geq 0}\left(\Delta_{\lambda} \otimes \mathrm{Id}\right) \Delta_{m}\left(-D^{\star}\right)^{(m+1)} .
\end{aligned}
$$

After replacing $\lambda$ by $-\lambda$, we compose (142) with $e^{D^{\star}(d / d \lambda)}$ to obtain

$$
\begin{aligned}
(\mathrm{Id} \otimes & \Lambda) \Delta_{-\lambda} e^{D^{\star}(d / d \lambda)} \\
= & \left(\Delta_{-\lambda} \otimes \mathrm{Id}\right) T \Lambda e^{D^{\star}(d / d \lambda)} \\
& +(T \otimes \mathrm{Id})\left(\mathrm{Id} \otimes \Delta_{-\lambda}\right) \Lambda e^{D^{\star}(d / d \lambda)} \\
& +\left(\int_{-D^{\star}}^{-\lambda}\left(\Delta_{-\lambda} \otimes \mathrm{Id}\right) \Delta_{\mu} d \mu\right) e^{D^{\star}(d / d \lambda)},
\end{aligned}
$$

where $e^{D^{\star}(d / d \lambda)}$ on the right indicates the position of the powers of $D^{\star}$ in the expansion. The powers of $(d / d \lambda)$ act on $\lambda$ as if they were placed on the left of each term, namely, as the derivation map. For instance, the expression $\Delta_{-\lambda} e^{D^{\star}(d / d \lambda)}$ is interpreted as follows:

$$
\Delta_{-\lambda} e^{D^{\star}(d / d \lambda)}=\sum_{n \geq 0} \sum_{k=0}^{n} \Delta_{n}\left(D^{\star}\right)^{(k)}\left(\frac{d}{d \lambda}\right)^{k}\left((-\lambda)^{(n)}\right) .
$$

Using (90) and (92) it follows that

$$
\begin{array}{r}
\Lambda e^{D^{\star}(d / d \lambda)}=\left(e^{D^{\star}(d / d \lambda)} \otimes e^{D^{\star}(d / d \lambda)}\right) \Lambda, \\
\Delta_{\lambda} e^{D^{\star}(d / d \lambda)}=\left(e^{D^{\star}(d / d \lambda)} \otimes e^{D^{\star}(d / d \lambda)}\right) \Delta_{\lambda} .
\end{array}
$$

Applying (146) and Taylor's Theorem, we obtain

$$
\begin{aligned}
&\left(\Delta_{-\lambda} \otimes \mathrm{Id}\right) T \Lambda e^{D^{\star}(d / d \lambda)} \\
&=\left(\Delta_{-\lambda} \otimes \mathrm{Id}\right) T \\
& \times\left(e^{D^{\star}(d / d \lambda)} \otimes e^{D^{\star}(d / d \lambda)}\right) \Lambda \\
&=\left(\Delta_{-\lambda-D^{\star}} \otimes e^{D^{\star}(d / d \lambda)}\right) T \Lambda, \\
&(T \otimes \mathrm{Id})\left(\mathrm{Id} \otimes \Delta_{-\lambda}\right) \Lambda e^{D^{\star}(d / d \lambda)} \\
&=(T \otimes \operatorname{Id})\left(e^{D^{\star}(d / d \lambda)} \otimes \Delta_{-\lambda-D^{\star}}\right) \Lambda .
\end{aligned}
$$

On the other hand, Taylor's Theorem implies

$$
\begin{gathered}
(\operatorname{Id} \otimes \Lambda) \Delta_{-\lambda} e^{D^{\star}(d / d \lambda)}=(\operatorname{Id} \otimes \Lambda) \Delta_{-\lambda-D^{\star}}, \\
\left(\int_{-D^{\star}}^{-\lambda}\left(\Delta_{-\lambda} \otimes \mathrm{Id}\right) \Delta_{\mu} d \mu\right) e^{D^{\star}(d / d \lambda)} \\
=\int_{-D^{\star}}^{-\lambda-D^{\star}}\left(\Delta_{-\lambda} \otimes \mathrm{Id}\right) \Delta_{\mu} e^{D^{\star}(d / d \lambda)} d \mu .
\end{gathered}
$$

Using conformal coskew symmetry (93) and making a change of variables we have

$$
\begin{aligned}
& \int_{-D^{\star}}^{-\lambda-D^{\star}}\left(\Delta_{-\lambda} \otimes \mathrm{Id}\right) \Delta_{\mu} e^{D^{\star}(d / d \lambda)} d \mu \\
& =-\int_{-D^{\star}}^{-\lambda-D^{\star}}\left(\Delta_{-\lambda} \otimes \mathrm{Id}\right) T \Delta_{-\mu-D^{\star}} e^{D^{\star}(d / d \lambda)} d \mu \\
& =\int_{0}^{\lambda}\left(\Delta_{-\lambda} \otimes \mathrm{Id}\right) T \Delta_{\mu} e^{D^{\star}(d / d \lambda)} d \mu .
\end{aligned}
$$

Using (92) it is easy to check that

$$
\begin{aligned}
T \Delta_{\mu} e^{D^{\star}(d / d \lambda)} & =T\left(\operatorname{Id} \otimes e^{\left(D^{\star}-\mu\right)(d / d \lambda)}\right) \Delta_{\mu} \\
& =\left(e^{\left(D^{\star}-\mu\right)(d / d \lambda)} \otimes \mathrm{Id}\right) T \Delta_{\mu} .
\end{aligned}
$$

Hence, using (151) together with (152) and (153), we obtain

$$
\begin{aligned}
& \left(\int_{-D^{\star}}^{-\lambda}\left(\Delta_{-\lambda} \otimes \mathrm{Id}\right) \Delta_{\mu} d \mu\right) e^{D^{\star}(d / d \lambda)} \\
& \quad=\int_{0}^{\lambda}\left(\Delta_{-\lambda} \otimes \mathrm{Id}\right)\left(e^{\left(D^{\star}-\mu\right)(d / d \lambda)} \otimes \mathrm{Id}\right) T \Delta_{\mu} d \mu,
\end{aligned}
$$

which due to Taylor's Theorem implies

$$
\begin{gathered}
\left(\int_{-D^{\star}}^{-\lambda}\left(\Delta_{-\lambda} \otimes \mathrm{Id}\right) \Delta_{\mu} d \mu\right) e^{D^{\star}(d / d \lambda)} \\
\quad=\int_{0}^{\lambda}\left(\Delta_{-\lambda+\mu-D^{\star}} \otimes \mathrm{Id}\right) T \Delta_{\mu} d \mu .
\end{gathered}
$$

Replacing (148), (149), (150), and (155) in (144) and using Taylor's Theorem we obtain

$$
\begin{aligned}
(\mathrm{Id} \otimes \Lambda) \Delta_{-\lambda-D^{\star}}= & \left(\Delta_{-\lambda-D^{\star}} \otimes e^{D^{\star}(d / d \lambda)}\right) T \Lambda \\
& +(T \otimes \mathrm{Id})\left(e^{D^{\star}(d / d \lambda)} \otimes \Delta_{-\lambda-D^{\star}}\right) \Lambda \\
& +\int_{0}^{\lambda}\left(\Delta_{-\lambda+\mu-D^{\star}} \otimes \mathrm{Id}\right) T \Delta_{\mu} d \mu .
\end{aligned}
$$

Next, using conformal coskew symmetry (93) we can rewrite (156) as follows:

$$
\begin{aligned}
(\mathrm{Id} \otimes \Lambda) T \Delta_{\lambda}= & \left(T \Delta_{\lambda} \otimes e^{D^{\star}(d / d \lambda)}\right) T \Lambda \\
& +(T \otimes \mathrm{Id})\left(e^{D^{\star}(d / d \lambda)} \otimes T \Delta_{\lambda}\right) \Lambda \\
& +\int_{0}^{\lambda}\left(T \Delta_{\lambda-\mu} \otimes \mathrm{Id}\right) T \Delta_{\mu} d \mu .
\end{aligned}
$$


Finally, it is easy to check that if we apply $(\mathrm{Id} \otimes T)(T \otimes I d)$ to (157), then we obtain (139).

(2) Cocompatibility (96) implies

$$
\begin{aligned}
(\Lambda \otimes & \operatorname{Id}) \Lambda-(\operatorname{Id} \otimes \Lambda) \Lambda \\
= & (\Lambda \otimes \operatorname{Id}) T \Lambda-(\operatorname{Id} \otimes T)(\operatorname{Id} \otimes \Lambda) \Lambda \\
& +\int_{-D^{\star}}^{0}(\Lambda \otimes \operatorname{Id}) \Delta_{\lambda} d \lambda \\
& -\left(\int_{\operatorname{Id} \otimes\left(-D^{\star}\right)}^{0}\left(\operatorname{Id} \otimes \Delta_{\lambda}\right) d \lambda\right) \Lambda .
\end{aligned}
$$

On the other hand, coleft symmetry (97) and cocompatibility (96) imply

$$
\begin{aligned}
(\Lambda & \otimes I \mathrm{Id}) T \Lambda-(\mathrm{Id} \otimes T)(\mathrm{Id} \otimes \Lambda) \Lambda \\
& =-(\mathrm{Id} \otimes T)(\Lambda \otimes \mathrm{Id}) \Lambda+(\mathrm{Id} \otimes T)(T \Lambda \otimes \mathrm{Id}) \Lambda \\
& =-(\mathrm{Id} \otimes T)\left(\int_{\left(-D^{\star}\right) \otimes \mathrm{Id}}^{0}\left(\Delta_{\lambda} \otimes \mathrm{Id}\right) d \lambda\right) \Lambda \\
& =-(T \otimes \mathrm{Id})\left(\int_{\mathrm{Id} \otimes\left(-D^{\star}\right)}^{0}\left(\operatorname{Id} \otimes \Delta_{\lambda}\right) d \lambda\right) T \Lambda .
\end{aligned}
$$

Using cocompatibility again on the right hand side of (159) we obtain

$$
\begin{aligned}
& (\Lambda \otimes I d) T \Lambda-(\operatorname{Id} \otimes T)(\operatorname{Id} \otimes \Lambda) \Lambda \\
& =-(T \otimes I d)\left(\int_{\operatorname{Id} \otimes\left(-D^{\star}\right)}^{0}\left(\operatorname{Id} \otimes \Delta_{\lambda}\right) d \lambda\right) \Lambda \\
& \quad+(T \otimes I d)\left(\int_{\operatorname{Id} \otimes\left(-D^{\star}\right)}^{0}\left(\operatorname{Id} \otimes \Delta_{\lambda}\right) d \lambda\right) \\
& \quad \times\left(\int_{-D^{\star}}^{0} \Delta_{\mu} d \mu\right) .
\end{aligned}
$$

Now, note that by using (92) it is clear that for $j \geq 0$

$$
\left(\operatorname{Id} \otimes\left(-D^{\star}\right)^{j}\right) \Delta_{\mu}=\Delta_{\mu}\left(-D^{\star}-\mu\right)^{j} .
$$

Therefore, the double integral in (160) satisfies

$$
\begin{aligned}
& \left(\int_{\operatorname{Id} \otimes\left(-D^{\star}\right)}^{0}\left(\operatorname{Id} \otimes \Delta_{\lambda}\right) d \lambda\right)\left(\int_{-D^{\star}}^{0} \Delta_{\mu} d \mu\right) \\
& \quad=\int_{-D^{\star}}^{0}\left(\int_{\operatorname{Id} \otimes\left(-D^{\star}\right)}^{0}\left(\operatorname{Id} \otimes \Delta_{\lambda}\right) d \lambda\right) \Delta_{\mu} d \mu \\
& \quad=\int_{-D^{\star}}^{0}\left(\int_{-D^{\star}-\mu}^{0}\left(\operatorname{Id} \otimes \Delta_{\lambda}\right) \Delta_{\mu} d \lambda\right) d \mu \\
& \quad=\int_{-D^{\star}}^{0}\left(\int_{-D^{\star}}^{\mu}\left(\operatorname{Id} \otimes \Delta_{\lambda-\mu}\right) \Delta_{\mu} d \lambda\right) d \mu \\
& \quad=\int_{-D^{\star}}^{0}\left(\int_{\lambda}^{0}\left(\operatorname{Id} \otimes \Delta_{\lambda-\mu}\right) \Delta_{\mu} d \lambda\right) d \lambda \\
& \quad=-\int_{-D^{\star}}^{0}\left(\int_{\lambda}^{0}\left(\operatorname{Id} \otimes \Delta_{\lambda-\mu}\right) \Delta_{\mu} d \lambda\right) d \lambda .
\end{aligned}
$$

Applying ( $T \otimes I d)$ to (162) and using (139), we obtain

$$
\begin{array}{r}
(T \otimes I d)\left(\int_{\operatorname{Id} \otimes\left(-D^{\star}\right)}^{0}\left(\operatorname{Id} \otimes \Delta_{\lambda}\right) d \lambda\right)\left(\int_{-D^{\star}}^{0} \Delta_{\mu} d \mu\right) \\
=-\int_{-D^{\star}}^{0}\left((\Lambda \otimes \mathrm{Id}) \Delta_{\lambda}-\left(e^{D^{\star}(d / d \lambda)} \otimes \Delta_{\lambda}\right) \Lambda\right. \\
\left.-(T \otimes I \mathrm{Id})\left(e^{D^{\star}(d / d \lambda)} \otimes \Delta_{\lambda}\right) \Lambda\right) d \lambda .
\end{array}
$$

Now, using (163) and (160) in (158) we have

$$
\begin{aligned}
& (\Lambda \otimes \operatorname{Id}) \Lambda-(\operatorname{Id} \otimes \Lambda) \Lambda \\
& =\int_{-D^{\star}}^{0}\left(e^{D^{\star}(d / d \lambda)} \otimes \Delta_{\lambda}\right) \Lambda d \lambda \\
& \quad-\left(\int_{\operatorname{Id} \otimes\left(-D^{\star}\right)}^{0}\left(\operatorname{Id} \otimes \Delta_{\lambda}\right) d \lambda\right) \Lambda \\
& \quad+(T \otimes \operatorname{Id}) \int_{-D^{\star}}^{0}\left(e^{D^{\star}(d / d \lambda)} \otimes \Delta_{\lambda}\right) \Lambda d \lambda \\
& \quad-(T \otimes \operatorname{Id})\left(\int_{\operatorname{Id} \otimes\left(-D^{\star}\right)}^{0}\left(\operatorname{Id} \otimes \Delta_{\lambda}\right) d \lambda\right) \Lambda .
\end{aligned}
$$

Note that by using (90) we can rewrite

$$
\begin{aligned}
& \int_{-D^{\star}}^{0}\left(e^{D^{\star}(d / d \lambda)} \otimes \Delta_{\lambda}\right) \Lambda d \lambda \\
& \quad=\left(\int_{-\left(D^{\star} \otimes I \mathrm{I}+\mathrm{I} \otimes D^{\star}\right)}^{0}\left(e^{D^{\star}(d / d \lambda)} \otimes \Delta_{\lambda}\right) d \lambda\right) \Lambda .
\end{aligned}
$$

Next, observe that Taylor's Theorem implies

$$
\begin{aligned}
& \int_{-\left(D^{\star} \otimes I \mathrm{~d}+\mathrm{Id} \otimes D^{\star}\right)}^{0}\left(e^{D^{\star}(d / d \lambda)} \otimes \Delta_{\lambda}\right) d \lambda \\
& =\int_{-\left(D^{*} \otimes \mathrm{Id}+\mathrm{Id} \otimes D^{\star}\right)}^{0} e^{\left(D^{*} \otimes \mathrm{Id}\right)(d / d \lambda)}\left(\operatorname{Id} \otimes \Delta_{\lambda}\right) d \lambda \\
& =\int_{-\left(D^{\star} \otimes \mathrm{Id}+\mathrm{I} d \otimes D^{*}\right)}^{0}\left(\mathrm{Id} \otimes \Delta_{\lambda+\left(D^{\star} \otimes \mathrm{Id}\right)}\right) d \lambda \\
& =\int_{-\left(D^{\star} \otimes \mathrm{Id}+\mathrm{Id} \otimes D^{\star}\right)}^{0} \sum_{k \geq 0}\left(\mathrm{Id} \otimes \Delta_{k}\right)\left(\lambda+\left(D^{\star} \otimes \mathrm{Id}\right)\right)^{(k)} d \lambda \\
& =\left.\sum_{k \geq 0}\left(\mathrm{Id} \otimes \Delta_{k}\right)\left(\lambda+\left(D^{\star} \otimes \mathrm{Id}\right)\right)^{(k+1)}\right|_{\lambda=-\left(D^{\star} \otimes \mathrm{I} \mathrm{d}+\mathrm{I} \mathrm{I} \otimes D^{\star}\right)} ^{\lambda=0} \\
& =\sum_{k \geq 0}\left(\mathrm{Id} \otimes \Delta_{k}\right)\left(\left(D^{\star}\right)^{(k+1)} \otimes \mathrm{Id}\right) \\
& -\sum_{k \geq 0}\left(\mathrm{Id} \otimes \Delta_{k}\right)\left(\mathrm{Id} \otimes\left(-D^{\star}\right)^{(k+1)}\right) \\
& =\int_{0}^{D^{\star} \otimes \mathrm{Id}}\left(\operatorname{Id} \otimes \Delta_{\lambda}\right) d \lambda+\int_{\operatorname{Id} \otimes\left(-D^{\star}\right)}^{0}\left(\operatorname{Id} \otimes \Delta_{\lambda}\right) d \lambda .
\end{aligned}
$$


Hence, in light of (165) and (166) we have

$$
\begin{aligned}
\int_{-D^{\star}}^{0} & \left(e^{D^{\star}(d / d \lambda)} \otimes \Delta_{\lambda}\right) \Lambda d \lambda \\
& -\left(\int_{\operatorname{Id} \otimes\left(-D^{\star}\right)}^{0}\left(\operatorname{Id} \otimes \Delta_{\lambda}\right) d \lambda\right) \Lambda \\
= & \left(\int_{0}^{D^{\star} \otimes \operatorname{Id}}\left(\operatorname{Id} \otimes \Delta_{\lambda}\right) d \lambda\right) \Lambda .
\end{aligned}
$$

Finally, using (167) in (164) it is clear that (140) holds, finishing the proof of Proposition 23.

Now, we can finish the proof of Theorem 21 . On the one hand, we have already proved that under the axioms of Definition 20, the $D^{\star}$ formula (52) holds. This implies that $\left(\left(D^{\star}\right)^{(i)} \otimes \mathrm{Id}\right) \Delta_{-1}=\Delta_{-1-i}$ for all $i \geq 0$, if we consider the expansion $\lambda(x)=\sum_{n \in \mathbb{Z}} \Delta_{n} x^{-n-1}$ with $\lambda(x)$ defined as in (117). Therefore, in the proof of Proposition 19(9) we proved that (110) is equivalent to (133) for $n=-1$ and $F=$ $z^{-1}$. In light of Proposition 23(2), it is clear that, under the axioms of Definition 20, that case holds. To finish the proof of Theorem 21, we need to show that (133) holds for $n=-1$ and $F=e^{\lambda z}$. We will prove that Proposition 23 (1) implies this last case.

First, note that considering $\lambda(z)$ as in (117), we have $\left(e^{D^{\star}(d / d \lambda)} \otimes \mathrm{Id}\right) \lambda(z)=e^{(d / d z)(d / d \lambda)} \lambda(z)$ due to the $D^{\star}$ formula (52) already proven to hold. This implies

$$
\begin{aligned}
(T & \otimes \mathrm{Id})\left(e^{D^{\star}(d / d \lambda)} \otimes \Delta_{\lambda}\right) \Lambda \\
& =\operatorname{Res}_{z} z^{-1}(T \otimes \mathrm{Id})\left(e^{D^{\star}(d / d \lambda)} \otimes \Delta_{\lambda}\right) \wedge(z) \\
& =\operatorname{Res}_{z} z^{-1} e^{(d / d z)(d / d \lambda)}(T \otimes \mathrm{Id})\left(\operatorname{Id} \otimes \Delta_{\lambda}\right) \wedge(z) \\
& =\operatorname{Res}_{z}(T \otimes \mathrm{Id})\left(\left(\operatorname{Id} \otimes \Delta_{\lambda-(d / d z)}\right)\left(z^{-1}\right)\right) \wedge(z),
\end{aligned}
$$

where the parenthesis indicate that $d / d z$ only acts on $z^{-1}$. Second, using that

$$
\left(\operatorname{Id} \otimes \Delta_{\lambda-\mu}\right) e^{\mu z}=\left(\operatorname{Id} \otimes \Delta_{\lambda-(d / d z)}\right) e^{\mu z},
$$

we have

$$
\begin{aligned}
& \int_{0}^{\lambda}(T \otimes I d)\left(\operatorname{Id} \otimes \Delta_{\lambda-\mu}\right) \Delta_{\mu} d \mu \\
& \quad=\int_{0}^{\lambda} \operatorname{Res}_{z}(T \otimes I d)\left(\operatorname{Id} \otimes \Delta_{\lambda-\mu}\right) e^{\mu z} \wedge(z) d \mu \\
& \quad=\operatorname{Res}_{z}(T \otimes I d)\left(\left(\operatorname{Id} \otimes \Delta_{\lambda-(d / d z)}\right)\left(\int_{0}^{\lambda} e^{\mu z} d \mu\right)\right) \wedge(z)
\end{aligned}
$$

Third, straightforward computation shows that

$$
\begin{aligned}
\left(e^{D^{\star}(d / d \lambda)} \otimes \Delta_{\lambda}\right) \Lambda & =\operatorname{Res}_{z}\left(e^{D^{\star}(d / d \lambda)} \otimes \lambda(z)\right) e^{\lambda z} \Lambda \\
& =\operatorname{Res}_{z} e^{\lambda z}\left(e^{z D^{\star}} \otimes(z)\right) \Lambda .
\end{aligned}
$$

Replacing (168), (170), and (171) in (139) and using that $z^{-1}+$ $\int_{0}^{\lambda} e^{\mu z} d \mu=e^{\lambda z} z^{-1}$, we obtain

$$
\begin{aligned}
\operatorname{Res}_{z}(\Lambda \otimes \mathrm{Id}) \wedge(z) e^{\lambda z} \\
=\operatorname{Res}_{z} e^{\lambda z}\left(e^{z D^{\star}} \otimes \lambda(z)\right) \Lambda+\operatorname{Res}_{z}(T \otimes \mathrm{Id}) \\
\quad \times\left(\left(\operatorname{Id} \otimes \Delta_{\lambda-(d / d z)}\right)\left(z^{-1}+\int_{0}^{\lambda} e^{\mu z} d \mu\right)\right) \wedge(z) \\
=\operatorname{Res}_{z} e^{\lambda z}\left(e^{z D^{\star}} \otimes \lambda(z)\right) \Lambda+\operatorname{Res}_{z}(T \otimes \mathrm{Id}) \\
\times\left(\left(\operatorname{Id} \otimes \Delta_{\lambda-(d / d z)}\right)\left(e^{\lambda z} z^{-1}\right)\right) \wedge(z) .
\end{aligned}
$$

Now, note that using that

$$
\left(\lambda-\frac{d}{d z}\right)^{n} e^{\lambda z}=e^{\lambda z}\left(-\frac{d}{d z}\right)^{n}
$$

we can rewrite the last term in (172) as follows:

$$
\operatorname{Res}_{z}(T \otimes \mathrm{Id})\left(\left(\mathrm{Id} \otimes \Delta_{-(d / d z)}\right)\left(z^{-1}\right)\right) \wedge(z) e^{\lambda z} .
$$

Next, we use (174) in (172) to obtain

$$
\begin{aligned}
\operatorname{Res}_{z}(\Lambda \otimes \mathrm{Id}) \wedge(z) e^{\lambda z} \\
=\operatorname{Res}_{z}\left[\left(e^{z D^{\star}} \otimes \wedge(z)\right) \Lambda+(T \otimes \mathrm{Id})\right. \\
\left.\quad \times\left(\left(\operatorname{Id} \otimes \Delta_{-(d / d z)}\right)\left(z^{-1}\right)\right) \wedge(z)\right] e^{\lambda z} .
\end{aligned}
$$

Finally, note that using the definition of $\lambda(z)$ in (117), it is easy to check that

$$
\begin{aligned}
& \left(e^{z D^{\star}} \otimes \wedge(z)\right) \Lambda+(T \otimes \operatorname{Id})\left(\left(\operatorname{Id} \otimes \Delta_{-(d / d z)}\right)\left(z^{-1}\right)\right) \wedge(z) \\
& \quad=\operatorname{cop}(-1)[\wedge(z)] .
\end{aligned}
$$

Therefore, replacing (176) in (175) we obtain

$$
\operatorname{Res}_{z}(\Lambda \otimes \mathrm{Id}) \wedge(z) e^{\lambda z}=\operatorname{Res}_{z} \operatorname{cop}(-1)[\lambda(z)] e^{\lambda z}
$$

which implies (133) for $n=-1$ and $F=e^{\lambda z}$, finishing the proof of Theorem 21 .

\section{Conflict of Interests}

The authors declare that there is no conflict of interests regarding the publication of this paper.

\section{Acknowledgments}

Florencia Orosz Hunziker was supported by a scholarship granted by Conicet, Consejo Nacional de Investigaciones Científicas y Técnicas, Argentina. José I. Liberati was supported in part by Conicet and ANPCyT. 


\section{References}

[1] K. Hubbard, "Vertex coalgebras, comodules, cocommutativity and coassociativity", Journal of Pure and Applied Algebra, vol. 213, no. 1, pp. 109-126, 2009.

[2] K. Hubbard, "Constructions of vertex operator coalgebras via vertex operator algebras," Journal of Algebra, vol. 294, no. 1, pp. 278-293, 2005.

[3] K. Hubbard, "The notion of vertex operator coalgebra and a geometric interpretation," Communications in Algebra, vol. 34, no. 5, pp. 1541-1589, 2006.

[4] K. Hubbard, "The duality between vertex operator algebras and coalgebras, modules and comodules," Contemporary Mathematics, vol. 442, pp. 339-354, 2007.

[5] R. E. Borcherds, "Vertex algebras, Kac-Moody algebras, and the Monster," Proceedings of the National Academy of Sciences of the United States of America, vol. 83, pp. 3068-3071, 1986.

[6] J. Lepowsky and H. Li, Introduction to Vertex Operator Algebras and Their Representations, vol. 227 of Progress in Mathematics, Birkhuser, Boston, Mass, USA, 2004.

[7] V. G. Kac, Vertex Algebras for Beginners, vol. 10 of University Lecture Series, American Mathematical Society, Providence, RI, USA, 1996, 2nd edition 1998.

[8] H. Li, "Local systems of vertex operators, vertex superalgebras and modules," Journal of Pure and Applied Algebra, vol. 109, no. 2, pp. 143-195, 1996.

[9] B. Bakalov and V. G. Kac, "Field algebras," International Mathematics Research Notices, no. 3, pp. 123-159, 2003.

[10] A. Matsuo and K. Nagatomo, Axioms for a Vertex Algebra and the Locality of Quantum Fields, vol. 4 of MSJ Memoirs, Mathematical Society of Japan, Tokyo, Japan, 1999.

[11] I. B. Frenkel, J. Lepowsky, and A. Meurman, Vertex Operator Algebras and the Monster, vol. 134 of Pure and Applied Mathematics, Academic Press, Boston, Mass, USA, 1988.

[12] M. Rosellen, "A course in vertexAlgebra," http://arxiv.org/abs/ math/0607270. 


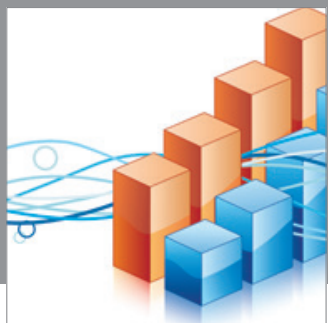

Advances in

Operations Research

mansans

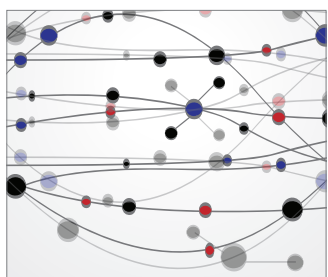

The Scientific World Journal
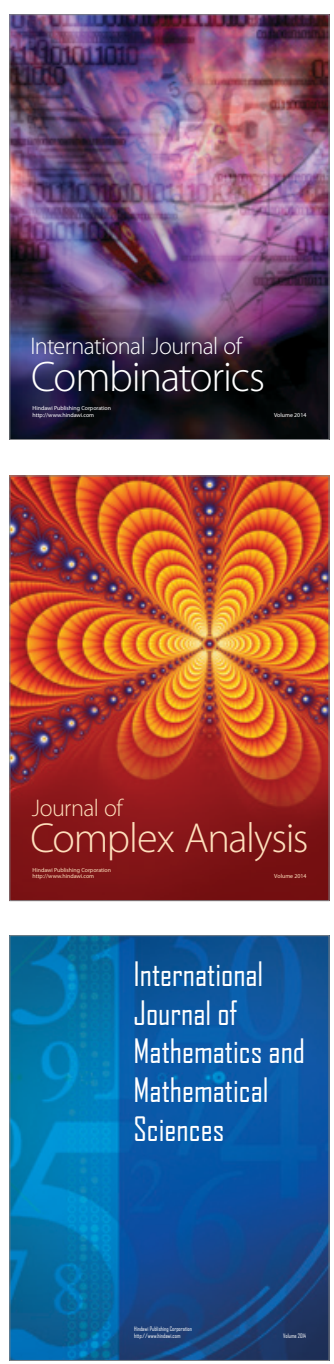
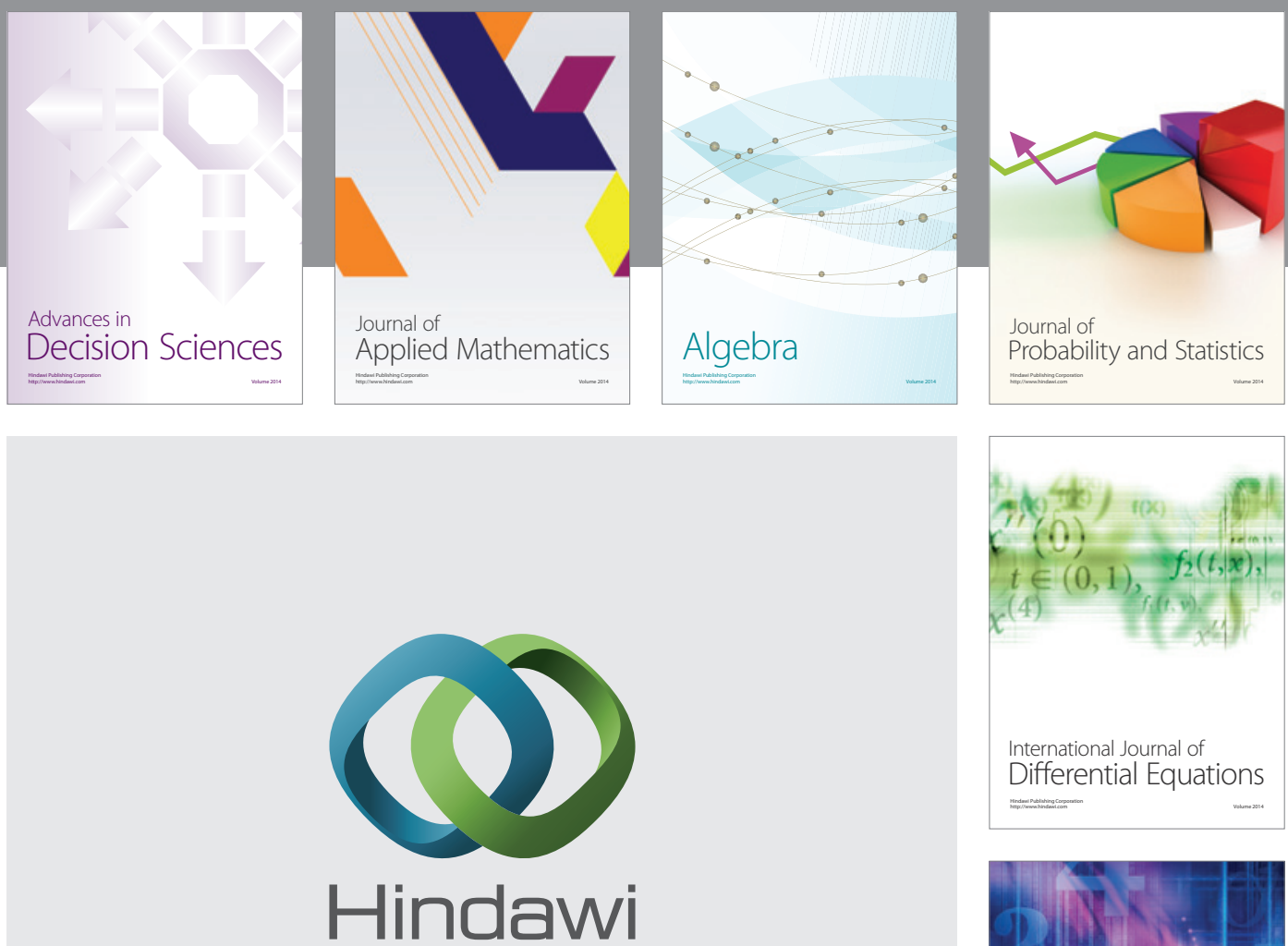

Submit your manuscripts at http://www.hindawi.com
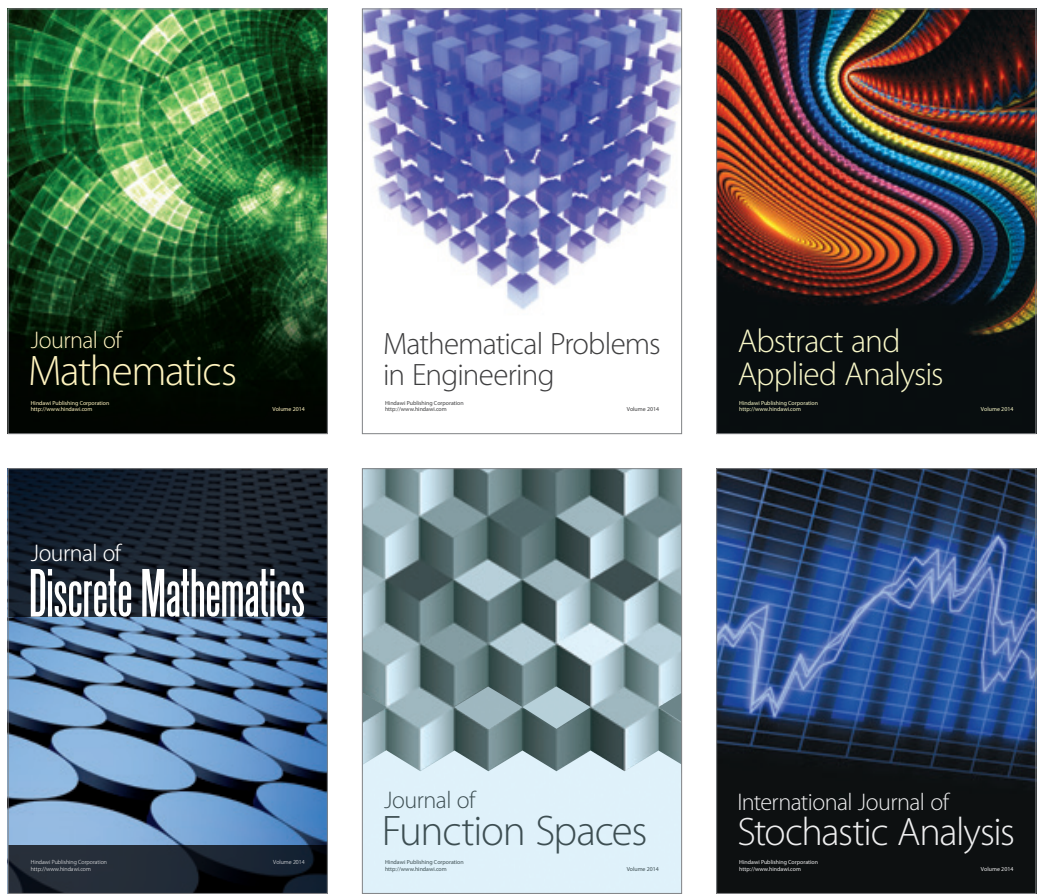

Journal of

Function Spaces

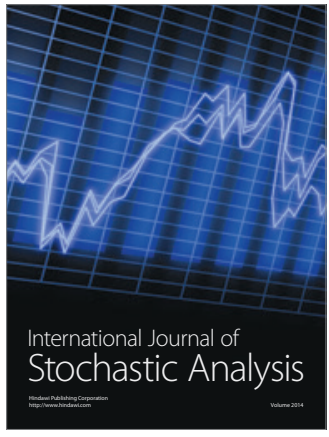

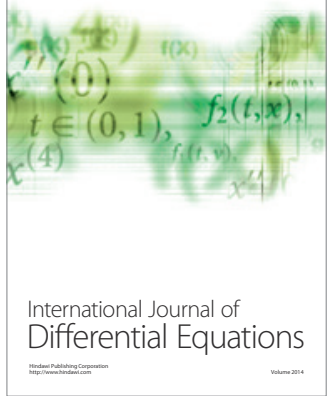
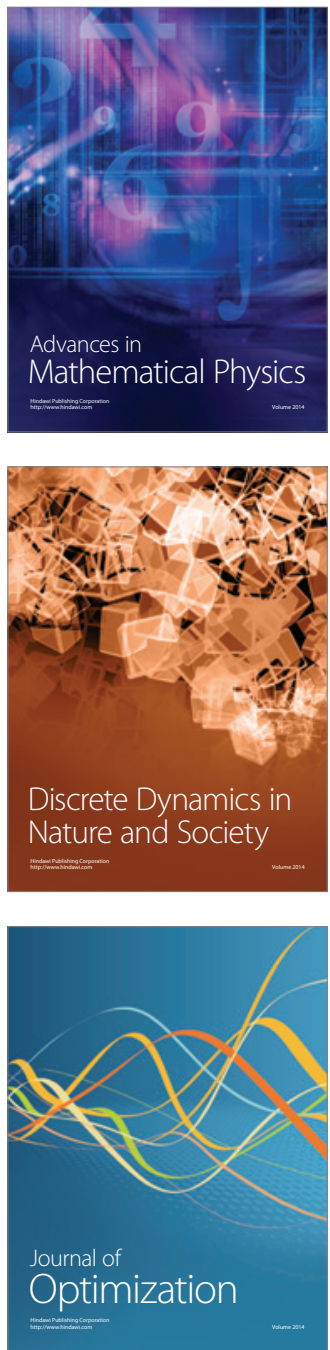